\title{
Inhibition of macrophage proliferation dominates plaque regression in response to cholesterol lowering
}

\author{
Carmen Härdtner ${ }^{1}$. Jan Kornemann ${ }^{1} \cdot$ Katja Krebs $^{1}$. Carolin A. Ehlert ${ }^{1}$ - Alina Jander ${ }^{1}$. Jiadai Zou ${ }^{1}$. \\ Christopher Starz $^{1}$ - Simon Rauterberg ${ }^{1}$. Diana Sharipova ${ }^{1}$ - Bianca Dufner ${ }^{1}$. Natalie Hoppe ${ }^{1} \cdot$ Tsai-Sang Dederichs $^{1}$. \\ Florian Willecke ${ }^{1}$. Peter Stachon ${ }^{1}$ - Timo Heidt ${ }^{1}$. Dennis Wolf ${ }^{1}$. Constantin von zur Mühlen ${ }^{1}$. Josef Madl ${ }^{2}$. \\ Peter Kohl ${ }^{2} \cdot$ Rafael Kaeser $^{3} \cdot$ Tobias Boettler $^{3}$ - Elsbeth J. Pieterman ${ }^{4} \cdot$ Hans M. G. Princen ${ }^{4} \cdot$ Benoît Ho-Tin-Noé ${ }^{5}$. \\ Filip K. Swirski ${ }^{6}$. Clinton S. Robbins ${ }^{7} \cdot$ Christoph Bode $^{1} \cdot$ Andreas Zirlik $^{1,8} \cdot$ Ingo Hilgendorf $^{1}$ (i)
}

Received: 6 September 2020 / Accepted: 1 December 2020 / Published online: 9 December 2020

(c) The Author(s) 2020

\begin{abstract}
Statins induce plaque regression characterized by reduced macrophage content in humans, but the underlying mechanisms remain speculative. Studying the translational APOE*3-Leiden.CETP mouse model with a humanized lipoprotein metabolism, we find that systemic cholesterol lowering by oral atorvastatin or dietary restriction inhibits monocyte infiltration, and reverses macrophage accumulation in atherosclerotic plaques. Contrary to current believes, none of (1) reduced monocyte influx (studied by cell fate mapping in thorax-shielded irradiation bone marrow chimeras), (2) enhanced macrophage egress (studied by fluorescent bead labeling and transfer), or (3) atorvastatin accumulation in murine or human plaque (assessed by mass spectrometry) could adequately account for the observed loss in macrophage content in plaques that undergo phenotypic regression. Instead, suppression of local proliferation of macrophages dominates phenotypic plaque regression in response to cholesterol lowering: the lower the levels of serum LDL-cholesterol and lipid contents in murine aortic and human carotid artery plaques, the lower the rates of in situ macrophage proliferation. Our study identifies macrophage proliferation as the predominant turnover determinant and an attractive target for inducing plaque regression.
\end{abstract}

Keywords Atherosclerosis $\cdot$ Macrophage $\cdot$ Proliferation $\cdot$ Plaque regression

\section{Introduction}

Carmen Härdtner and Jan Kornemann contributed equally.

Supplementary Information The online version contains supplementary material available at https://doi.org/10.1007/s0039 5-020-00838-4.

Ingo Hilgendorf

ingo.hilgendorf@universitaets-herzzentrum.de

1 Department of Cardiology and Angiology I, University Heart Center Freiburg-Bad Krozingen and Faculty of Medicine, University of Freiburg, 55 Hugstetter St, 79106 Freiburg, Germany

2 Institute for Experimental Cardiovascular Medicine, University Heart Center Freiburg-Bad Krozingen and Faculty of Medicine, University of Freiburg, Freiburg, Germany

3 Department of Medicine II, Faculty of Medicine, Medical Center-University Freiburg, University of Freiburg, Freiburg, Germany
International guidelines recommend treating patients with atherosclerotic disease with high-dose statins [20, 41]. For every $40 \mathrm{mg} / \mathrm{dL}$ reduction in low-cholesterol diet (LDL) cholesterol by statins the relative risk for major adverse

4 The Netherlands Organization for Applied Scientific Research (TNO)-Metabolic Health Research, Leiden, Netherlands

5 INSERM Unit 1148, University Paris Diderot, and Laboratory for Vascular Translational Science, Sorbonne Paris Cité, Paris, France

6 Center of Systems Biology, Massachusetts General Hospital and Harvard Medical School, Boston, MA, USA

7 Peter Munk Cardiac Centre, University Health Network, Toronto, Canada

8 Department of Cardiology, University of Graz, Graz, Austria 
cardiovascular events is curtailed by about $20 \%$ [17]. Intravascular imaging studies of atherosclerotic coronaries documented plaque regression with a reduction in plaque volume and macrophage content alongside an increase in fibrous cap thickness following statin treatment [24].

Statins lower LDL-cholesterol levels by inhibiting hydroxy-methylglutaryl-coenzyme A reductase-dependent cholesterol biosynthesis and inducing LDL receptor expression in the liver. LDL-cholesterol lowering also reduces $\mathrm{C}$-reactive protein. These and other findings have fueled the debate as to whether the beneficial effects of statins are primarily attributed to lipid lowering or to pleiotropic, i.e. anti-inflammatory and directly vasoprotective, effects [23, 40]. Multiple in vitro studies documented lipid-independent effects of statins on macrophages and endothelial cells, and statin treatment of Apolipoprotein E (Apoe)-deficient mice limited atherogenesis and monocyte recruitment without affecting cholesterol levels [3, 21, 51, 69]. In contrast, APOE*3Leiden.Cholesteryl ester transfer protein (CETP) mice, a translational mouse model with a humanized lipoprotein metabolism [70], respond to oral statin treatment with (V)LDL-cholesterol lowering, and showed attenuated plaque formation when fed a Western diet [25]. The authors proposed impaired monocyte recruitment into atherosclerotic lesions and suppressed inflammation as underlying mechanisms [26, 64].

While recruited monocytes give rise to plaque macrophages, we showed that local macrophage proliferation dominates cell renewal in established lesions in atherosclerotic mice [49]. Macrophage proliferation in atherosclerotic lesions, particularly in foam cell rich areas, has been described before $[19,45,50]$, but its relevance to plaque development was not known. When monocyte production and recruitment are attenuated experimentally, new onset atherogenesis is limited, while interventions in established disease fail to slow plaque progression as lesional macrophages continue to proliferate [29]. Although plaque regression is the ultimate goal in cardiovascular preventive medicine, controversy still surrounds the mechanisms that control the decline in plaque macrophages. Reduction in monocyte influx $[13,42]$, requirement of differentiation of infiltrating monocytes into reparative macrophages [43], and macrophage emigration $[16,30]$ were reported to determine plaque regression. In this study, we examined the relative contribution of monocyte infiltration, macrophage proliferation, death and egress in APOE*3Leiden.CETP mice, which model human-like lipid changes in response to oral statin treatment. We show that monocyte-to-macrophage differentiation is a relatively rare event, both in plaque progression and regression, and that the decline in macrophage numbers in regressing plaques mainly results from the suppression of cholesterol-driven local proliferation. Notably, plaque lipid contents and serum cholesterol levels in patients undergoing carotid endarterectomy positively correlated with local macrophage proliferation, supporting the rationale for targeting macrophage proliferation therapeutically.

\section{Methods}

\section{Animals and diet}

Female APOE*3-Leiden.CETP mice were purchased from TNO (Leiden, Netherlands). We used female mice as they are more susceptible to cholesterol-containing diets by having higher plasma cholesterol levels relative to APOE*3-Leiden.CETP males, with established dose-dependent responses to statins $[63,70]$. Correspondingly, we purchased female Apoe $^{-/-}$mice (B6.129P2-Apoe ${ }^{\text {tmlUnc }}$ ) from The Jackson Laboratory (Bar Harbor, ME, USA). 8-week-old mice were fed a high-cholesterol diet (HCD, 1.25\% w/w cholesterol, D12108 mod., Ssniff GmBH, Soest, Germany) ad libitum for 12 weeks to accelerate atherogenesis, followed by a low-cholesterol diet (LCD, 0.05\% w/w cholesterol, semi-synthetic diet T, TNO, Leiden) for 4 weeks to lower proatherogenic plasma cholesterol to levels that allow for normalization through intervention. Next, mice were randomly assigned to up to three groups: 1 . continued LCD (control group), 2 . LCD supplemented with $0.01 \% \mathrm{w} / \mathrm{w}$ atorvastatin (Pfizer) corresponding to $10 \mathrm{mg} / \mathrm{kg}$ body weight per day (statin group), and 3. diet $\mathrm{T}$ without cholesterol (free group) for another 4 weeks. To translate the dosing used in our mouse studies $(10 \mathrm{mg} / \mathrm{kg} / \mathrm{d})$ to human dosing, the following simplified calculation based on body surface area, as accepted by the FDA, can be used as a guide: mouse dose/ $12.3 \times$ human body weight [36]. Accordingly, $10 \mathrm{mg} / \mathrm{kg} /$ day atorvastatin in mice correspond to $65 \mathrm{mg} / \mathrm{day}$ for an $80 \mathrm{~kg}$ human.

Female $\mathrm{Ldlr}^{--}$mice (B6.129S7-Ldlr ${ }^{\mathrm{tm} 1 \mathrm{Her}} / \mathrm{J}$ ) were lethally irradiated $(10 \mathrm{~Gy})$ and reconstituted with a 1:1 mixture of bone marrow cells from CD45.1 C57B1/6 (B6.SJL-Ptprc ${ }^{\mathrm{a}}$ $\left.\mathrm{Pepc}^{\mathrm{b}} / \mathrm{BoyJ}\right)$ and CD45.2 $\mathrm{Msr}^{-/-}\left(\mathrm{B} 6 . \mathrm{Cg}-\mathrm{Msr}^{\left.\mathrm{tm}^{1 \mathrm{Csk}} / \mathrm{J}\right) \text { or }}\right.$ CD45.2 $\mathrm{CD}^{-1-}\left(\mathrm{B} 6.129 \mathrm{~S} 1-\mathrm{CD} 36^{\mathrm{tm} 1 \mathrm{Mfe} / \mathrm{J}}\right)$ mice, as previously described [49]. Mice were purchased from The Jackson Laboratory (Bar Harbor, ME, USA). Following 6 weeks of reconstitution consuming chow diet, mice were fed $\mathrm{HCD}$ (1.25\% w/w cholesterol, D12108 mod., Ssniff GmBH, Soest, Germany) for 12 weeks to induce atherosclerosis.

Mice were housed under specific pathogen-free conditions

\section{Patients}

Twenty-three patients scheduled for elective carotid endarterectomy because of significant carotid artery stenosis gave written informed consent to having their blood and endarterectomy specimens analyzed as approved by the Institutional 
Review Board of the University Hospital of Freiburg. Blood and tissue samples were collected within $12 \mathrm{~h}$ after the last intake of statin drugs if applicable. Detailed patient characteristics are listed in supplemental table 1. Liver tissue biopsies were sampled from three patients during bariatric surgery that had been on oral atorvastatin $40-80 \mathrm{mg} /$ day. Detailed patient characteristics are listed in supplemental table 2 .

\section{In vivo cell labeling}

Four weeks into the high-cholesterol diet, 1- $\mu \mathrm{m}$ large yellow-green fluorescent beads (Fluoresbrite YG plain microspheres, Polysciences Inc., Eppelheim, Germany), diluted in 1:4 sterile PBS, were injected intravenously into $\mathrm{APOE}^{*} 3$ Leiden.CETP and Apoe ${ }^{-/-}$mice, respectively, for in vivo cell labeling and tracking of myeloid cells. Fluorescent bead accumulation in the plaque was quantified by immunofluorescence histology. $1 \mathrm{mg}$ bromodeoxyuridine (BrdU) (BD Bioscience, San Jose, CA, USA) was injected intravenously $2 \mathrm{~h}$ prior to killing to label proliferating macrophages. BrdU incorporation into $\mathrm{CD} 68^{+}$macrophages in the plaque was quantified by immunofluorescence histology.

\section{Partial body irradiation and bone marrow transfer}

Female UBC-GFP mice (C57BL/6-Tg(UBC-GFP)30Scha/J) were purchased from The Jackson Laboratory (Bar Harbor, ME, USA) as donors for bone marrow cell transplantation. APOE*3Leiden.CETP mice with established atherosclerosis following 11 weeks of HCD were lethally irradiated with 10 Gy (RS-2000 Pro Biological System; Rad Source, Buford, GA, USA) while shielding the torso with a lead casing (Belly Shield, Braintree Scientific Inc, Braintree MA, USA, placed on a $1 / 4$-inch-thick lead plate) to protect the thoracoabdominal aorta from radiation. $1 \times 10^{7} \mathrm{GFP}^{+}$bone marrow cells were intravenously transplanted into partially irradiated mice.

Modified LDL uptake by bone marrow-derived M $\phi$ $2 \times 10^{5}$ bone marrow cells were isolated from CD45.1 C57Bl/6 wild-type (WT), CD45.2 $\mathrm{Msr}^{-/-}$and CD45.2 $\mathrm{CD} 36^{-1-}$ mice which were used to generate mixed irradiation bone marrow chimeras in $\mathrm{Ldll}^{-/-}$, as described above. Bone marrow cells differentiating into bone marrow-derived $\mathrm{M} \phi(\mathrm{BMDM})$ in the presence of $30 \mathrm{ng} / \mathrm{ml}$ colony stimulating factor 1 (Peprotech, Rocky Hill, NJ, USA) over 5 days were stimulated with DiI-medium oxidized LDL $(10 \mu \mathrm{g} / \mathrm{ml})$, DiI-acetylated LDL $(1 \mu \mathrm{g} / \mathrm{ml})$ (Kalen Biomedical, Montgomery Village, MD, USA) or PBS for $4 \mathrm{~h}$ before being detached from 24-well plates using trypsin/EDTA. Cells were stained with fixable viability dye (Thermofisher Scientific, Waltham, MA, USA) and anti-F4/80 (Biolegend, San Diego, CA, USA) for flow cytometric quantification of percent viable BMDM and mean fluorescence intensity (MFI) of DiI-labeled modified LDL into the respective cells.

\section{Histology}

Murine aortic roots and arches were embedded in Optimal cutting temperature (OCT) Tissue Tek (Sakura Finetek, Tokyo, Japan) and cut into serial cryostat sections $(5 \mu \mathrm{m})$ starting at the level of the aortic valve (for root sections). Sections were stained with Oil-red O (Sigma-Aldrich, St. Louis, MO, USA), Masson Trichrome (Sigma-Aldrich, St. Louis, MO, USA), TUNEL (DeadEnd ${ }^{\mathrm{TM}}$ Fluorometric TUNEL System, Promega, Mannheim, Germany), antiCD68 (clone FA-11, BioRad AbD Serotec, Puchheim, Germany), anti-BrdU (GTX128091, GeneTex, Irvine, CA, USA and ab1893, Abcam, Cambridge, UK), anti-GFP (ab290, Abcam, Cambridge, UK). Human carotid endarterectomy specimens were embedded in OCT, frozen, sectioned, permeabilized with $0.1 \%$ Triton X-100, and stained with with anti-Ki67 (ab15580, Abcam, Cambridge, UK), anti-CD68 (clone PG-M1, Agilent, Santa Clara, CA, USA), and Hoechst 33342 (Thermofisher Scientific, Waltham, MA, USA). Adjacent slides of the same plaques were stained for Oilred O (Sigma-Aldrich, St. Louis, MO, USA). Secondary antibodies included rabbit-anti rat biotin conjugated (BA4001) followed by ImmPACT AMEC Red Substrate (Vector Laboratories, Burlingame, CA, USA), rabbit-anti rat TRITC (PA1-28570, Thermofisher Scientific, Waltham, MA, USA), rabbit-anti rat AF647 (ab169349, Abcam, Cambridge, UK), goat-anti-rabbit (BA-1000) followed by fluorescein avidin DCS (A-2011, Vector Laboratories, Burlingame, CA, USA), donkey-anti-sheep TRITC (ab6897, Abcam, Cambridge, UK), alpaca-anti-rabbit AF488 (ChromoTek, Planegg-Martinsried, Germany), and DAPI Mounting Medium (Carl Roth, Karlsruhe, Germany) according to the manufacturers' instructions. Images were recorded with the Axioplan 2 and Apotome 2 imaging light-/fluorescence microscope with an AxioCam camera (Carl Zeiss MicroImaging GmbH, Göttingen, Germany) and a confocal Leica TCS SP8 X microscope (Leica Microsystems, Wetzlar, Germany). Images were analyzed with Image Pro Premiere 9.2 (Media Cybernetics, Silver Springs, USA) and Zeiss Zen lite (Carl Zeiss MicroImaging $\mathrm{GmbH}$, Göttingen, Germany).

\section{Flow cytometry}

Murine aortic cells were retrieved through enzymatic digestion with collagenase I, collagenase XI, hyaluronidase, DNAse I and HEPES solution (Sigma-Aldrich, St. Louis, MO, USA) in a thermocycler for $45 \mathrm{~min}$ at $750 \mathrm{rpm}$ and $37^{\circ} \mathrm{C}$. Murine blood samples were lysed in RBC lysis buffer (Biolegend, San Diego, CA, USA). Isolated cells from the blood and aorta were counted using a Neubauer chamber 
(Marienfeld, Lauda-Königshofen, Germany). Cells were stained with specific fluorescent antibodies as indicated (Supplemental Table 3). Ly6C $\mathrm{C}^{\text {high }}$ monocytes were identified as $\mathrm{CD} 45^{+} \mathrm{CD} 11 \mathrm{~b}^{+}, \mathrm{Lin}^{-}(\mathrm{Lin}=\mathrm{CD} 3, \mathrm{CD} 19, \mathrm{NK} 1.1, \mathrm{Ly} 6 \mathrm{G})$, Ly6C ${ }^{\text {high }}, \mathrm{CD}_{115^{+}}, \mathrm{F} 4 / 80^{\text {low }}$. Macrophages were identified as $\mathrm{CD} 45^{+} \mathrm{CD}_{11 b^{+}}, \mathrm{Lin}^{-}, \mathrm{Ly} 6 \mathrm{C}^{\text {low }}, \mathrm{F} 4 / 80^{\text {high }}$. Intracellular staining with anti-Ki67 and anti-active Caspase 3, BD Cytoxfix/Cytoperm (\#554,722, BD Biosciences, San Diego, CA, USA), BD Perm/Wash (\#554,723) and BD Permeabilization Buffer Plus $(\# 561,651)$ was conducted according to the manufacturer's instructions. Data were collected on a BD Facs Canto II (BD Bioscience, San Diego, CA, USA) and analyzed with FlowJo (Treestar, Ashland, OR, USA).

\section{Real-time polymerase chain reaction (PCR)}

RNA was extracted from murine aortas using Qiazol and RNeasy Mini Kit (Qiagen, Valencia, CA, USA) according to the manufacturer's instructions. Quantitative TaqManPCR was performed using a Bio-Rad CFX96 Touch RealTime PCR System and TaqMan probes Mm00443258_m1 (Tnfa), Mm01336189_m1 (IL1ß), Mm00446190_m1 (IL6), Mm00439614_m1 (IL10), Mm01178820_m1 (Tgfb1), Mm01320970_m1 (Vcam1), Mm00441242_m1 (Ccl2). Data were statistically analyzed using the $2^{-\Delta \mathrm{Ct}}$ method.

\section{Lipid and enzyme-linked immunosorbent assays}

Murine plasma cholesterol and triglyceride levels were measured using cholesterol and triglycerides FS 10' Multipurpose kits (DiaSys Diagnostic Systems GmbH, Holzheim, Germany) according to the manufacturer's instructions. Lipoprotein profiling in murine plasma samples was conducted by LipoSEARCH services (Skylight Biotech Inc., Akita, Japan). Serum Amyloid A levels were measured using a SAA Mouse ELISA Kit (Thermo Fisher Scientific, Waltham, MA, USA), and ApoB levels were measured using the Mouse ApoB ELISA Kit (Abcam, Cambridge, UK) according to the manufacturers' instructions.

\section{Mass spectrometry}

Plasma and tissue samples were weighed, snap frozen and sent to ImaBiotech SAS (Lille, France) for the quantification of atorvastatin concentration by mass spectrometry. In brief, matrix-assisted laser desorption ionization-Fourier transform ion cyclotron resonance (MALDI-FTICR) was used for imaging mass spectrometry analysis of atorvastatin in murine frozen liver samples sectioned onto indium-tin-oxide glass slides and covered with DAN MALDI matrix $(10 \mathrm{mg} / \mathrm{ml})$ in acetonitrile:water 1:1 $(\mathrm{v} / \mathrm{v})$. Recordings were made using MALDI-FTICR in negative ionization mode and CASI (continuous accumulation of selected ions) mode centered on $\mathrm{m} / \mathrm{z} 564.2917 \pm 50 \mathrm{Da}$ mass range with a laser set at 300 shots, $1 \mathrm{kHz}$ to follow atorvastatin in the sections at $200 \mu \mathrm{m}$ spatial resolution with a SolariX mass spectrometer (Bruker Daltonics, Bremen, Germany). FTMS Control 2.0 and FlexImaging 4.1 software packages (Bruker Daltonics, Bremen, Germany) were used to control the mass spectrometer and set imaging parameters. For liquid tomography-tandem mass spectrometry (LC-MS/ MS)-based quantification of atorvastatin, murine tissue samples were extracted with methanol, and murine plasma and human samples were extracted with acetonitrile, and spiked with the internal standard atorvastatin-d5, $1.5 \mathrm{nM}$. The LC-MS/MS consisted of an UHPLC Ultimate $3000 \mathrm{cou}-$ pled with TSQ Quantiva (Thermo Scientific, Courtaboeuf, France) equipped with a Cortecs C18 $2.7 \mu \mathrm{m} ; 2.1 \times 30 \mathrm{~mm}$ column (Waters, Saint-Quentin-en-Yvelines, France).

\section{Statistics}

Results are presented as mean \pm SEM. Differences between two groups were analyzed with unpaired Student's $T$ test or Mann-Whitney test as indicated in the figure legend. To assess differences between more than two groups, one-way ANOVA with Holm-Sidak's multiple comparisons testing or Kruskal-Wallis with Dunn's multiple comparisons testing were applied. $p$ values $\leq 0.05$ denote significant changes. Pearson's correlation coefficient was used to test for correlation.

\section{Results}

\section{Oral atorvastatin and diet-induced cholesterol lowering induce phenotypic plaque regression}

To study mechanisms of statin-mediated plaque regression, we induced atherosclerosis in APOE*3-Leiden.CETP mice by feeding a $1.25 \%$ cholesterol diet (high-cholesterol diet, HCD) over 12 weeks, accelerating plaque formation, followed by 4 weeks of $0.05 \%$ cholesterol diet (low-cholesterol diet, LCD) to lower plasma cholesterol levels to about $10 \mathrm{mmol} / \mathrm{L}$, that would allow for lipid normalization by subsequent therapeutic intervention. At 16 weeks, a baseline group was sacrificed and the remaining mice were randomized to continued LCD that allows for moderate plaque progression, LCD supplemented with $0.01 \%$ atorvastatin or a diet free of cholesterol. After 4 weeks, both atorvastatin and the cholesterol-free diet had cut plasma cholesterol levels by half, reaching normolipidemic starting levels. At this point, the three study groups were sacrificed for comparative analysis (Fig. 1a, b). Notably, the $10 \mathrm{mg} / \mathrm{kg} / \mathrm{d}$ atorvastatin dose in APOE*3-Leiden.CETP mice decreased total and non-HDL cholesterol levels by about $50 \%$, similar to the 
a
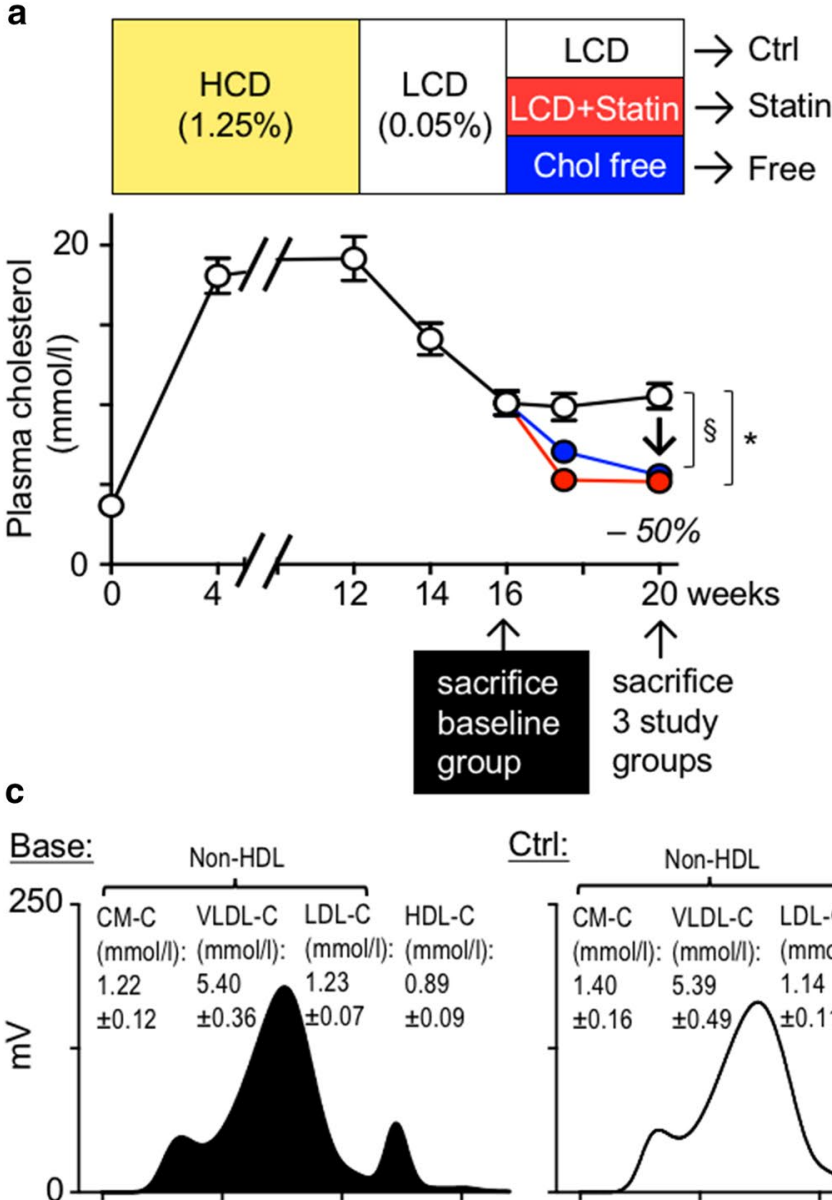

Statin:
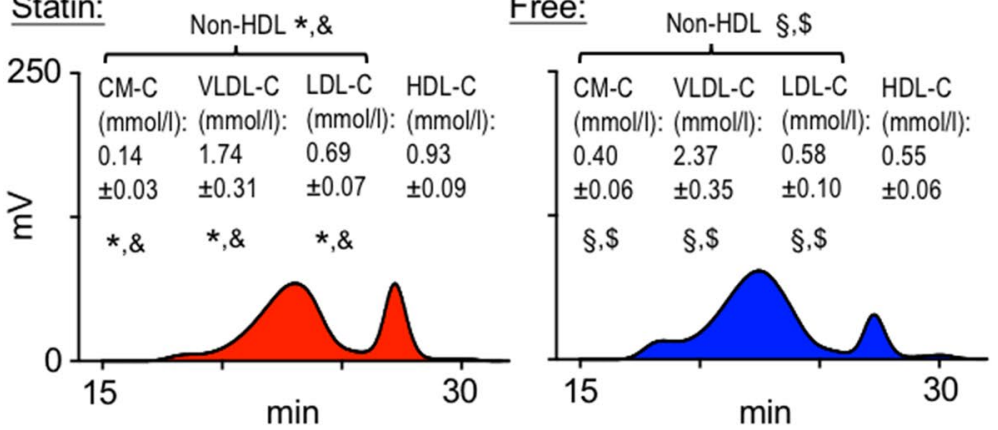

b
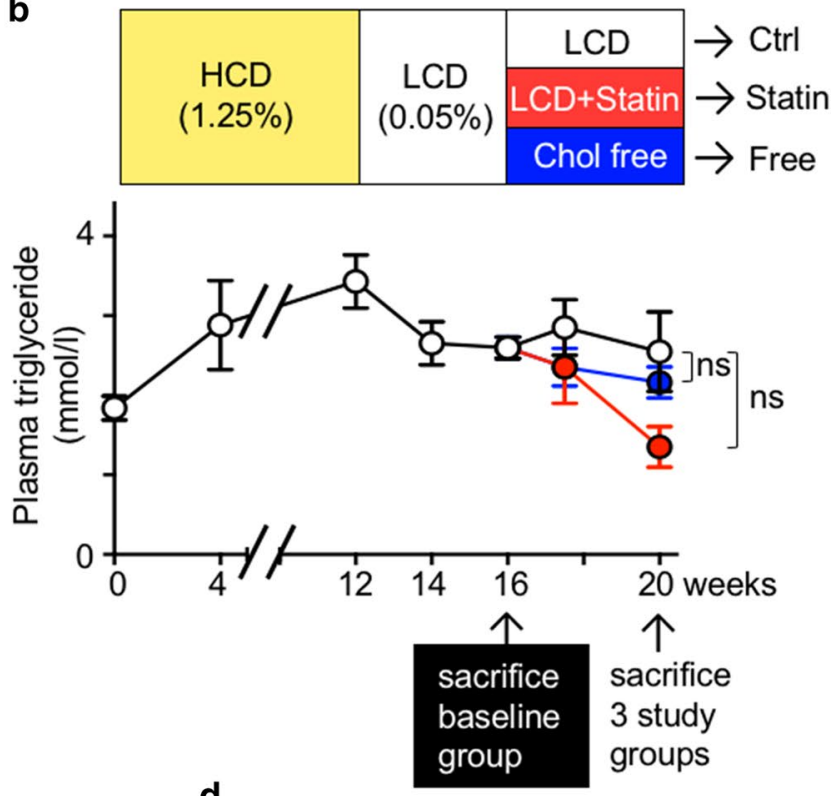

d
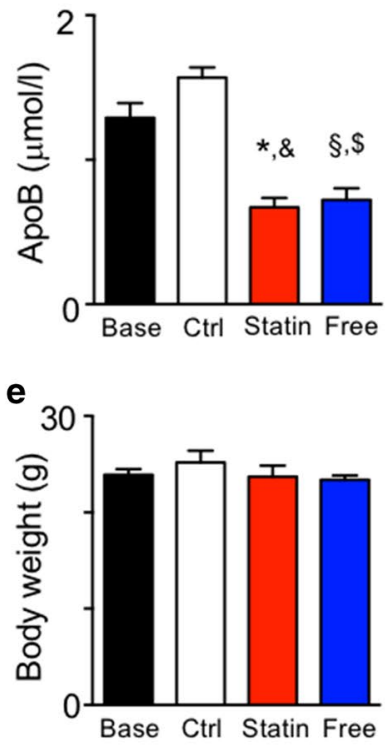

Fig. 1 Experimental plaque regression study using atorvastatin and cholesterol-free diet. a, b Study design and diet scheme depicting plasma total cholesterol (a) and triglyceride (b) levels measured while feeding APOE*3-Leiden.CETP mice a high-cholesterol diet (HCD, 1.25\% w/w cholesterol) for 12 weeks, followed by 4 weeks of low-cholesterol diet (LCD, 0.05\% w/w cholesterol). At 16 weeks, the baseline group (Base) was sacrificed and the remaining mice were randomized to three study groups, continued LCD (control, Ctrl), LCD supplemented with $0.01 \%$ (w/w) atorvastatin (Statin), and a diet free of cholesterol (Free) for another 4 weeks. Data are presented as mean \pm SEM. *, $\$ p<0.05$ denote statistically significant differences between the Control and Statin $(*)$ or Control and Free (§) groups at the time of killing (week 20), $n=6$ per group, one-way ANOVA. c Representative chromatograms and quantification of cholesterol in 4 major lipoprotein classes by gel-filtration HPLC. Data are presented as mean \pm SEM. ${ }^{*}, \&, \S, \$ p<0.05$ denote statistically significant differences between the Base and Statin (\&) or Base and Free (\$) groups, and between Control and Statin $(*)$ or Control and Free $(\S)$ groups at the time of sacrifice, $n=6$ per group, one-way ANOVA. d ApoB plasma levels at the time of sacrifice. Results are presented as mean \pm SEM, ${ }^{*}, \&, \S, \$ p<0.05$ denote statistically significant differences between the Base and Statin (\&) or Base and Free (\$) groups, and between Control and Statin $(*)$ or Control and Free $(\S)$ groups at the time of sacrifice, $n=6$ per group, one-way ANOVA. e Final body weight in all 4 groups at time of killing (Base $n=6, n=8$ other groups) 
effects observed in humans taking atorvastatin 40-80 mg per day [53] (Fig. 1a, c). Moreover, atorvastatin or dietary cholesterol restriction significantly reduced circulating ApoB levels (Fig. 1d), a quantitative marker of atherogenic lipoprotein particles, in analogy to changes observed in patients treated with oral atorvastatin [27, 58]. Triglyceride levels fluctuated modestly over the course of diet changes and did not differ between the study groups, unlike cholesterol levels (Fig. 1b). Body weights were similar in all groups (Fig. 1e).

Atherosclerotic lesion size was quantified in the aortic root and arch. Adding atorvastatin to LCD, or feeding a diet free of cholesterol, prevented plaque growth and altered plaque composition. While continued LCD increased lipid and macrophage contents and decreased collagen deposition, atorvastatin and cholesterol-free diets reversed these changes. We refer to these compositional changes as phenotypic plaque regression (Fig. 2a; Supplemental Figs. 1 and 2). As reported previously [64], reducing cholesterol levels by atorvastatin or diet suppresses serum amyloid A levels in plasma, indicative of anti-inflammatory effects (Fig. 2b). Specifically, IL- $1 \beta$ and IL-6 expressions are reduced, and TGF $\beta$ is increased in atherosclerotic aortas (Fig. 2c).

\section{Oral atorvastatin limits monocyte recruitment and macrophage accumulation in atherosclerotic aortas}

Quantification of macrophage numbers in enzymatically digested atherosclerotic aortas confirmed a reduction by more than $42 \%$ in response to atorvastatin treatment or cholesterol-free diet compared to LCD controls (Supplemental Fig. 3a). Concomitantly, aortic Ly6C ${ }^{\text {high }}$ monocyte counts were reduced significantly in atorvastatin-treated mice, and tended to be lower in mice fed a diet free of cholesterol with aortic VCAM-1 and CCL2 expression levels being suppressed (Supplemental Fig. 3a-c). Since the numbers of circulating blood monocytes were similar in all groups we conclude that cholesterol lowering by atorvastatin or dietary restrictions impairs both monocyte infiltration and macrophage accumulation in atherosclerotic lesions (Supplemental Fig. 3a, b and Supplemental Fig. 7a).

Next, we asked whether these two processes were causally linked. To this end, we complemented the feeding and treatment scheme in mice by lethal irradiation before the end of the HCD feeding while shielding the torso to protect the thoracoabdominal aorta from irradiation (Fig. 3a, b). By doing so, $\mathrm{M} \phi$ that had already accumulated in atherosclerotic plaques following 11 weeks of HCD feeding would not be affected by irradiation, preserving natural turnover kinetics. We then transplanted green fluorescent protein $(\mathrm{GFP})^{+}$bone marrow cells, which seeded in the irradiated, non-protected areas of the body, giving rise to $\mathrm{GFP}^{+}$monocytes and their progeny as a tool to quantify recruitment-dependent and recruitment-independent contributions to $\mathrm{M} \phi$ accumulation in established plaques. One week later, the diet was switched as before to LCD for 4 weeks (Fig. 3a). Meanwhile, a stable GFP cell chimerism of $17.3 \pm 2.3 \%$ established in circulating Ly6C ${ }^{\text {high }}$ monocytes (Fig. 3d,e; Supplemental Fig. 4c). The baseline reference group was killed at week 16 and studied, while the remaining mice were randomized to continued LCD, LCD $+0.01 \%$ atorvastatin, or cholesterol-free diet. After 4 weeks of treatment, these three study groups were killed at week 20, and blood and aortas were analyzed with regard to lesion development and GFP cell chimerism. As in the original model (Fig. 1a), both atorvastatin treatment and cholesterol-free diet reduced plasma cholesterol levels by about $50 \%$ compared to continued LCD (control) in chimeric mice, while body weights and blood Ly6 $\mathrm{C}^{\text {high }}$ monocyte numbers did not differ between the groups (Fig. 3a; Supplemental Fig. 4a, b). Likewise, histological evaluation of atherosclerotic lesions in the aortic roots and arches replicated the previously observed regression phenotype in irradiated, bone marrow transplanted mice, treated with atorvastatin or fed a diet free of cholesterol (Fig. 3c; Supplemental Figs. 4 and 5). The GFP cell chimerism in Ly6 $\mathrm{C}^{\text {high }}$ monocytes was similar in the blood and aortic tissue of all four groups, indicating that circulating $\mathrm{GFP}^{+}$monocytes infiltrated the atherosclerotic aortas, and that monocyte chimerism remained at equilibrium during the 4-week treatment period (Fig. 3d, e). A GFP chimerism of $15 \%$ in aortic Ly6Chigh monocytes means that for every $\mathrm{GFP}^{+}$monocyte recruited to the aorta, 6 to $7 \mathrm{GFP}^{-}$endogenous monocytes infiltrated as well $(100 \% / 15 \%=6.7)$. If all macrophages in the plaque renewed through replacement by infiltrating monocytes during the 4 weeks of plaque progression (weeks 16 to 20), GFP chimerism among monocytes should translate 1:1 into GFP chimerism among macrophages. However, this was not the case. GFP chimerism in aortic macrophages was only $1 / 10$ of the chimerism in aortic Ly6 $\mathrm{C}^{\text {high }}$ monocytes at baseline (week 16) (Fig. 3d), and although it increased significantly, it did so only by an absolute $1.7 \pm 0.6 \%$ following 4 weeks of plaque progression with continued LCD (week 20) (Fig. 3e). To estimate the relative contribution of all recruited monocytes to the aortic macrophage pool during plaque progression, accounting for both $\mathrm{GFP}^{+}$and $\mathrm{GFP}^{-}$Ly6C ${ }^{\text {high }}$ monocytes that infiltrated, the $1.7 \%$ absolute increase in $\mathrm{GFP}^{+}$ macrophages over 4 weeks of LCD needs to be multiplied by factor 6.7 , resulting in about $11 \%$ (Fig. 3f). Although the increase in recruited monocyte-derived macrophages was blunted by atorvastatin treatment in our model of established atherosclerosis, a reduction in macrophage numbers exceeding $11 \%$, such as the $>42 \%$ reduction observed with atorvastatin treatment compared to LCD controls (Supplemental Fig. 3a), will mainly depend on local processes of macrophage accumulation that are distinct from and additional to monocyte recruitment and differentiation. Low 

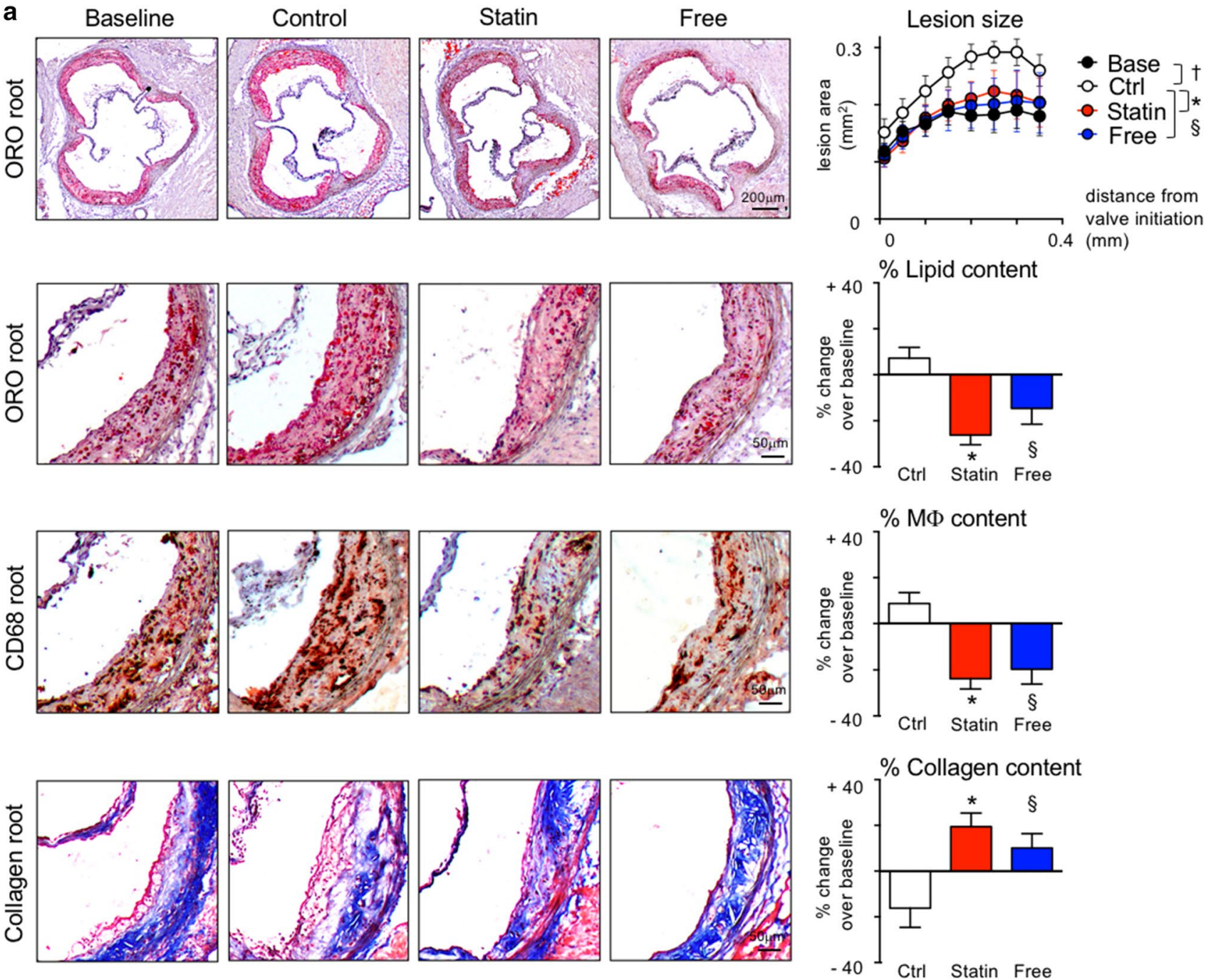

b Serum amyloid $\mathrm{A}$ c

$\mathrm{IL}-1 \beta$

IL-6

$\mathrm{TNF} \alpha$

TGF $\beta$
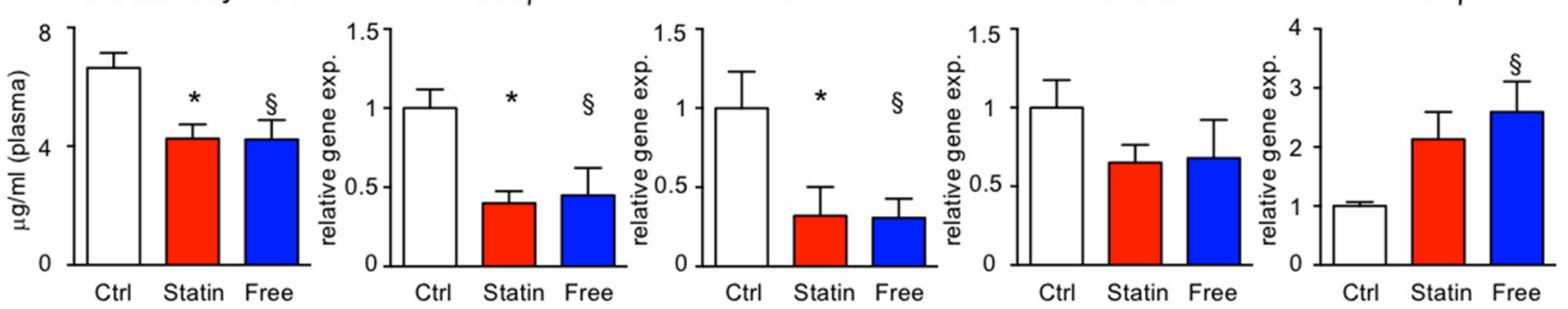

Fig. 2 Serum cholesterol lowering leads to phenotypic plaque regression in APOE*3-Leiden.CETP mice. a Representative images of aortic root sections stained with Oil-red O (ORO), anti-CD68 (for macrophages, $\mathrm{M} \phi$ ) and Masson's Trichrome (for collagen) on the left. On the right, lesion area was measured on 8 aortic root sections at $50 \mathrm{~m}$ intervals starting from valve initiation. Plaque composition was analyzed by quantifying the percent $\mathrm{ORO}^{+}, \mathrm{CD}^{+} 8^{+}$and collagen ${ }^{+}$areas within lesions. Data are presented as mean \pm SEM percent change over values in the baseline group to visualize relative changes during plaque progression and regression. $*, \S, \dagger p<0.05$ denote statistically significant differences between the Ctrl and Base $(\dagger)$, Statin $(*)$ and Free (§) groups, respectively, baseline $n=6$, other 3 groups $n=8$ per group, one-way ANOVA. (b) Serum amyloid A plasma levels at the time of sacrifice. Results are presented as mean \pm SEM, $*, \$ p<0.05$ denote statistically significant differences between the Ctrl and Statin $(*)$ or Free (§) groups, $n=8$ per group, one-way ANOVA. c Cytokine expression in atherosclerotic aortas. Results are presented as mean \pm SEM fold change over control group values, $*, \$ p<0.05$ denote statistically significant differences between the Ctrl and Statin (*) or Free (§) groups, $n=8$ per group, one-way ANOVA 
a
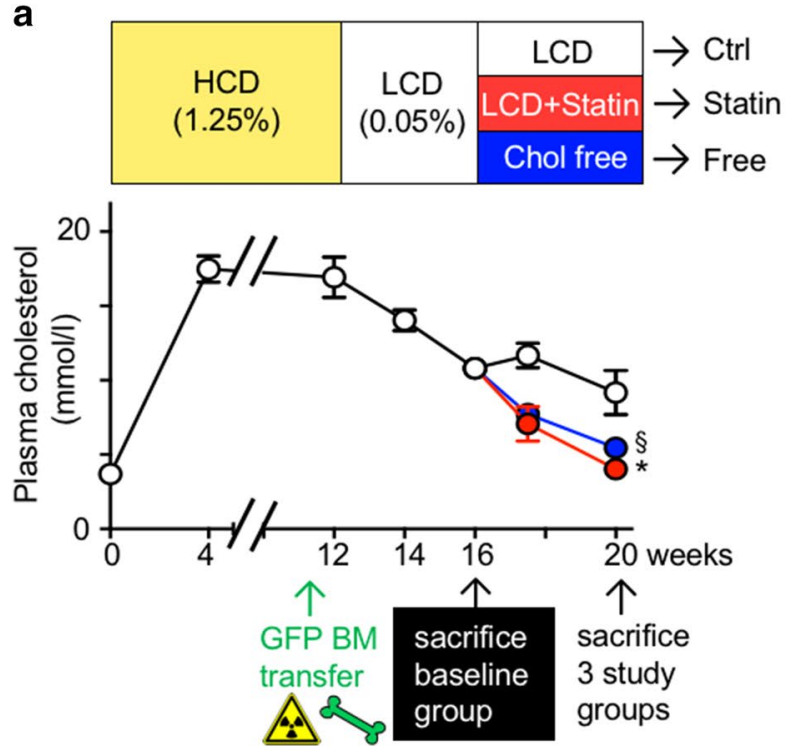

d Blood Lin- CD11b+ Blood Ly6Chigh mono

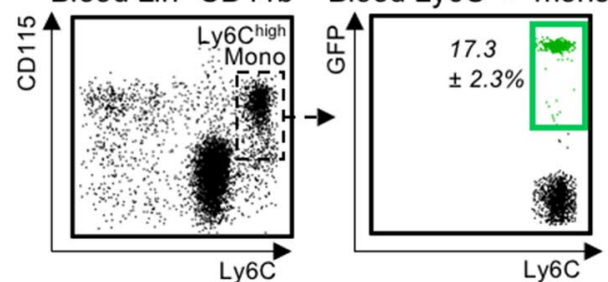

Aortic Lin- CD11 b+ Aortic Ly6C high mono
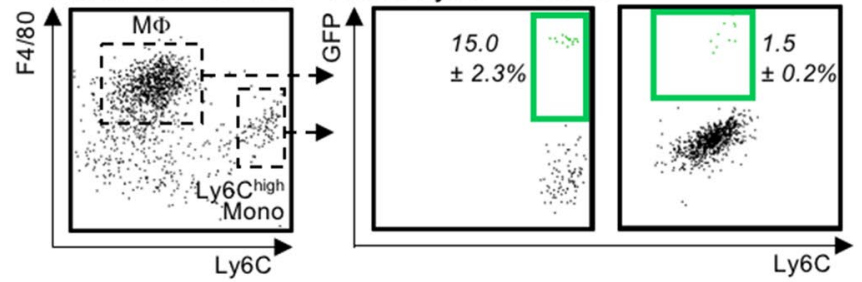

e
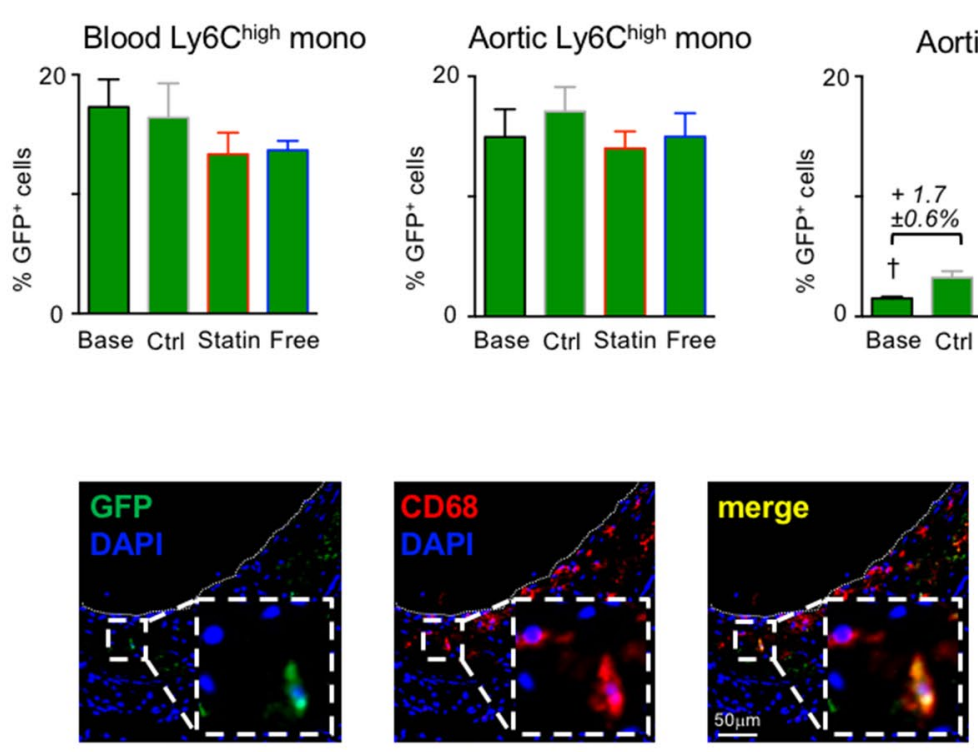

g
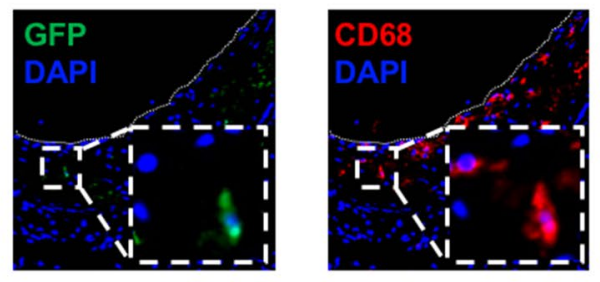

b

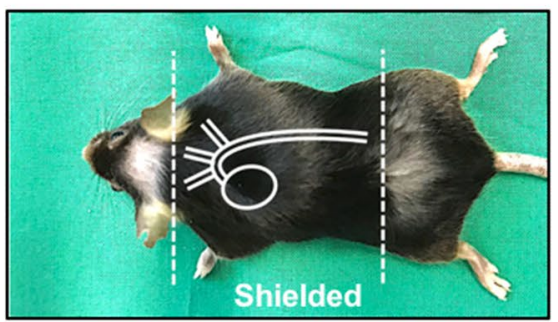

c

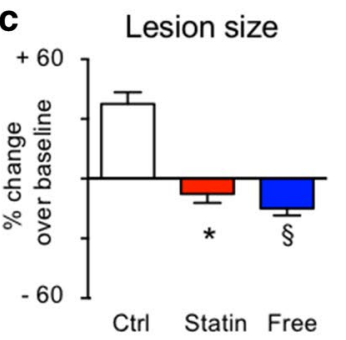

$\%$ Lipid content

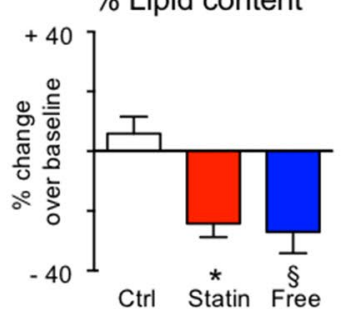

\% M $\Phi$ content

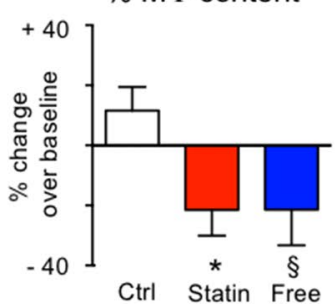

$\%$ Collagen content

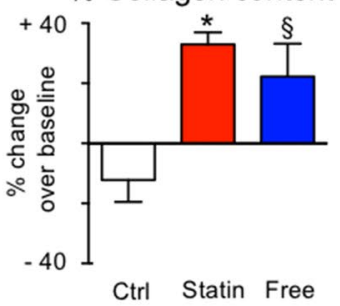

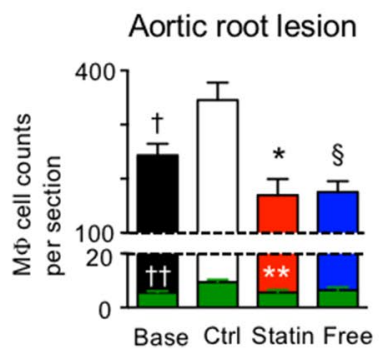


४Fig. 3 Serum cholesterol lowering reduces lesional macrophage accumulation in established disease, mostly independent of monocyte infiltration in APOE*3-Leiden.CETP mice. a, b Study design and diet scheme in analogy to Fig. 1a, modified by APOE*3-Leiden. CETP mice undergoing lethal irradiation at week 11 of HCD feeding while shielding the heart and aorta $\mathbf{b}$ with lead followed by GFP+ bone marrow cell transplantation. Plasma cholesterol levels at indicated time points are presented as mean \pm SEM. *, $\S p<0.05$ denote statistically significant differences between the Ctrl and Statin (*) or Free (§) groups at the time of sacrifice (week 20), $n=8$ per group and time point, one-way ANOVA. c Quantification of atherosclerotic lesion size and plaque composition in the aortic root. Data are presented as mean \pm SEM percent change over values in the baseline group to visualize relative changes during plaque progression and regression. ${ }^{*}, \S p<0.05$ denote statistically significant differences between the Ctrl and Statin $(*)$ or Free ( $§$ ) groups, baseline $n=6$, all other 3 groups $n=8$ per group, one-way ANOVA. Representative images are shown in Supplemental Fig. 4d. d Representative images of flow cytometric dot plots showing CD11b+ leukocytes in the aorta and blood at baseline, 5 weeks after GFP+ bone marrow cell transplantation. Data for GFP chimerism among Ly6Chigh monocyte and $\mathrm{M} \phi$ are presented as mean \pm SEM. e Flow cytometry based quantification of the GFP chimerism among Ly6Chigh monocytes and macrophages $(\mathrm{M} \phi)$ in the blood and aortic cell suspensions, respectively, in all four groups. Results are presented as mean \pm SEM, $*, \dagger$ $p<0.05$ denote statistically significant differences between the Ctrl and Base $(\dagger)$ or Statin (*) groups, $n=6$ (baseline) and $n=8$ all other groups, one-way ANOVA. f Estimation of the Ly6Chigh monocyte contribution to the pool of aortic M $\phi$ based on the changes in GFP chimerism during plaque progression (weeks 16-20). g Immunofluorescence histology-based quantification of GFP+ (green) cells among all $\mathrm{CD} 68+\mathrm{M} \phi$ in aortic root lesions on the right, and representative images of GFP, CD68 and DAPI co-stainings on the left. Results are presented as mean $\pm \mathrm{SEM}, *, \S, \dagger p<0.05$ denote statistically significant differences in all CD68+ M $\phi$ cell numbers between the Ctrl and Statin $(*)$, Free $(\S)$ or Base $(\dagger)$ groups. $* *, \dagger \dagger p<0.05$ denote statistically significant differences in GFP+ CD68+ M $\phi$ cell numbers between the Ctrl and Base ( $\dagger$ ) or Statin (**)groups, $n=6-8$ per group, one-way ANOVA. Representative images for all groups are shown in Supplemental Fig. 4d

frequencies of recruited $\mathrm{GFP}^{+} \mathrm{CD}^{+} 8^{+}$macrophages within established atherosclerotic lesions, and the decline in overall macrophage numbers in response to cholesterol lowering, were independently confirmed by histology (Fig. 3g; Supplemental Figs. 4d and 5).

\section{Oral atorvastatin limits plaque macrophage accumulation predominantly by reducing local macrophage proliferation}

We previously reported that local macrophage proliferation plays a dominant role during plaque progression [49], but its relevance for plaque regression has remained uncertain. Other local processes of cell turnover, such as cell death and egress, were described to influence macrophage content in atherosclerotic lesions [32]. Given that monocyte influx contributes minimally to plaque macrophage kinetics in our model of established atherosclerosis with plaque regression, we decided to compare the frequencies of macrophage proliferation, death and egress in the four study groups. Bromodeoxyuridine (BrdU), a thymidine analog that readily integrates into newly synthesized DNA during the cell cycle, was injected $2 \mathrm{~h}$ prior to killing. During this short time period, newly generated monocytes in the bone marrow will not have yet entered blood circulation, and they will, therefore, not contribute to the $\mathrm{BrdU}^{+}$fraction of macrophages in the plaque. Under these conditions, BrdU incorporation into plaque macrophages indicates local proliferation [49]. The proportion of proliferating macrophages was quantified based on immunofluorescent co-staining of anti-BrdU with DAPI and anti-CD68 in aortic root and arch lesions. Macrophage proliferation was reduced to nearly half of timematched control levels when adding atorvastatin to LCD or switching to cholesterol-free diet (Fig. 4a; Supplemental Figs. 2, 4d, 5, 6a). These data were independently confirmed by flow cytometric intracellular staining for Ki67 in aortic macrophages (Fig. 4b; Supplemental Fig. 7b). Macrophage death was quantified based on TUNEL co-staining with DAPI and anti-CD68 in aortic root and arch lesions, and flow cytometric intracellular staining for active caspase 3 (Fig. 4b, c; Supplemental Figs. 2 and 7b). We observed no difference between the groups in either test. Potential macrophage egress was determined using fluorescent bead labeling, as previously described [16, 29, 55, 67]. Fluorescent beads were injected early during atherogenesis after 4 weeks of HCD feeding (Supplemental Fig.6d). Within 7 days, these beads were cleared from the blood stream by circulating monocytes and neutrophils (Supplemental Fig. 6e) that infiltrate the nascent plaques. Since monocytes and neutrophils are short lived, beads that do not immediately leave the plaque within the cells that brought them in remain inside monocyte-derived macrophages. Alternatively, beads are disposed and trapped in the plaque when bead-laden cells die, until being taken up by other phagocytes. Only as beadladen phagocytes emigrate from the plaque, the number of beads inside the plaque can decrease over time. Therefore, the total number of beads that are retained in the plaques is inversely proportional to the number of macrophages that might exit the plaque. We did not observe a significant decline in plaque bead content over the 4-week time period of plaque regression (Fig. 4d; Supplemental Figs. 2 and 6c), indicating that macrophage egress is unlikely to be a major contributor to the loss of macrophages with oral atorvastatin treatment. Taken together, our data show that suppression of local macrophage proliferation in the presence of unchanged cell death mediates atorvastatin induced plaque regression.

\section{Reduced macrophage proliferation depends on cholesterol lowering in mice}

We wondered whether the observed effects of oral atorvastatin treatment were primarily mediated by cholesterol 
a

Aortic root lesion

Prolif. MФ
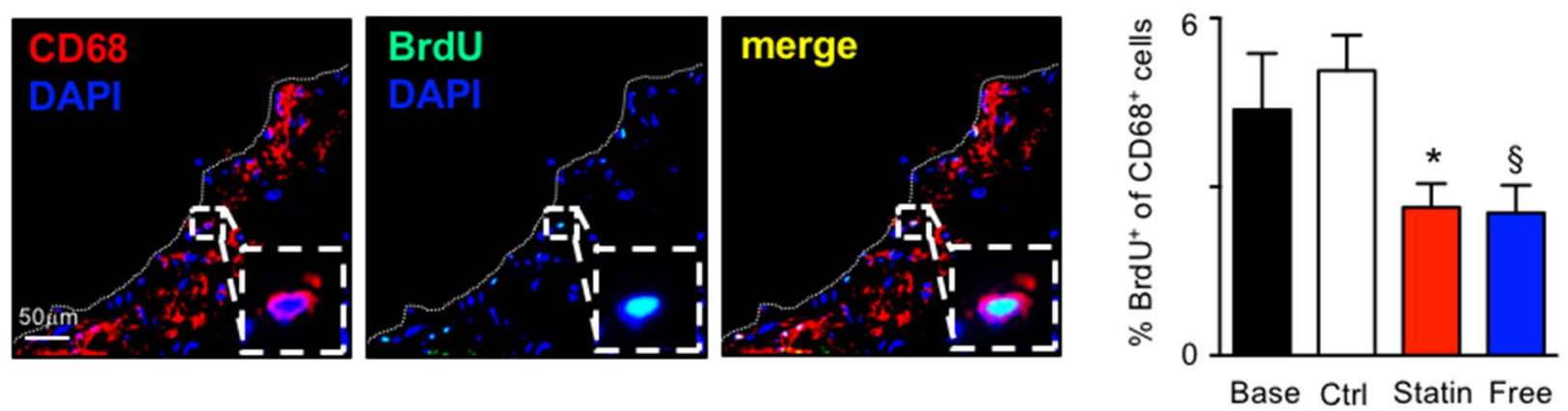

b Aortic Lin- CD11b+ leukocytes
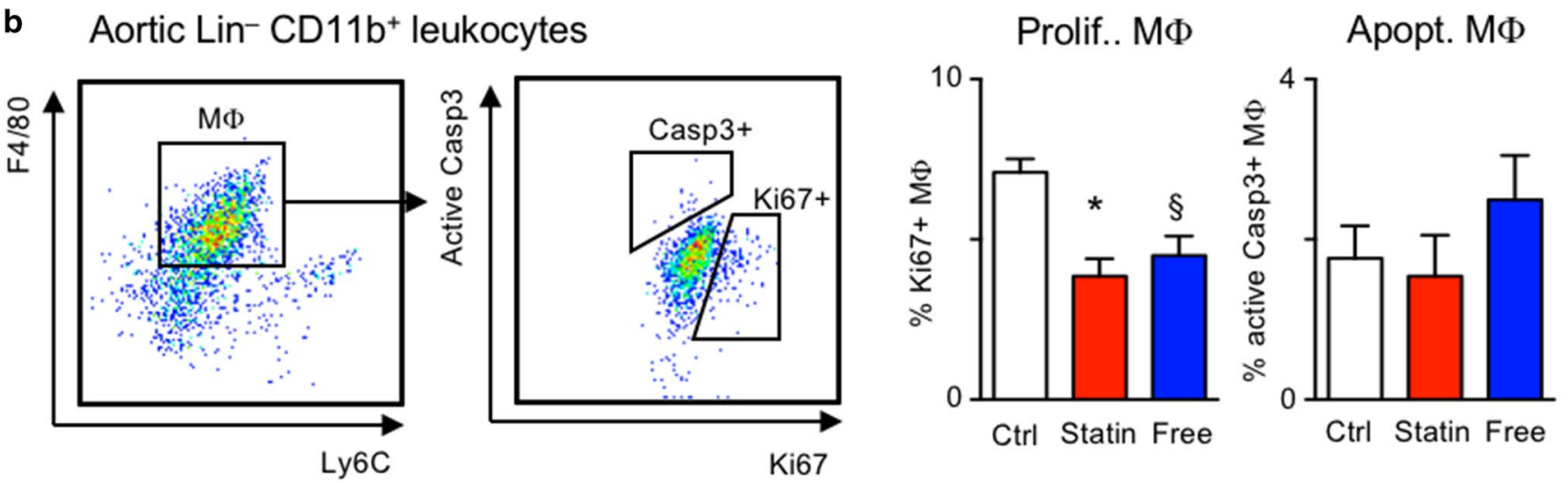

c Aortic root lesion

Apopt. МФ
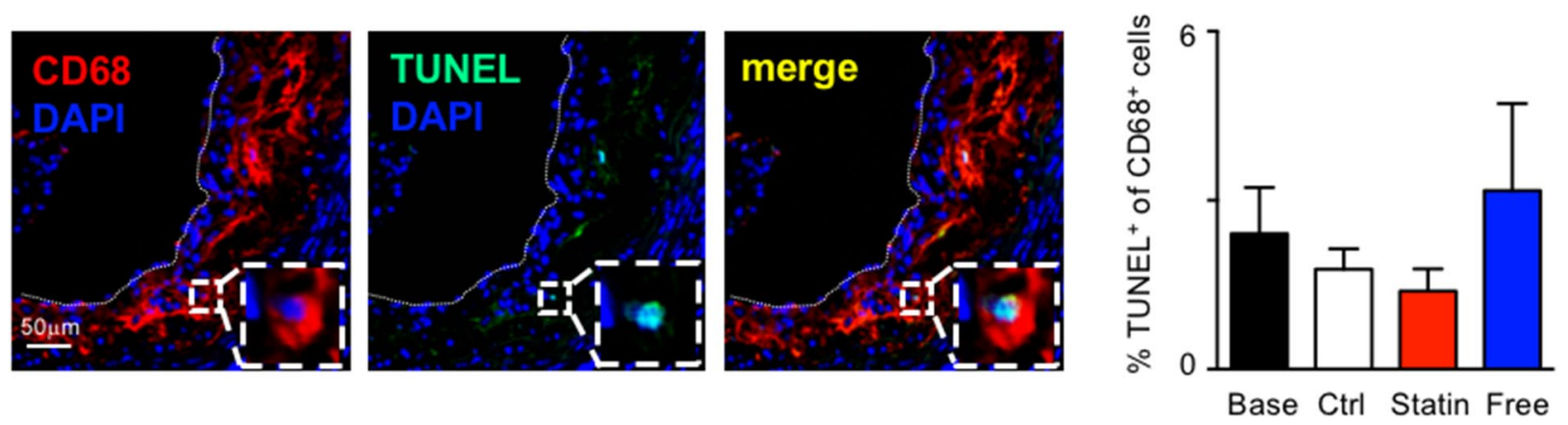

d Aortic root lesion

Beads per lesion
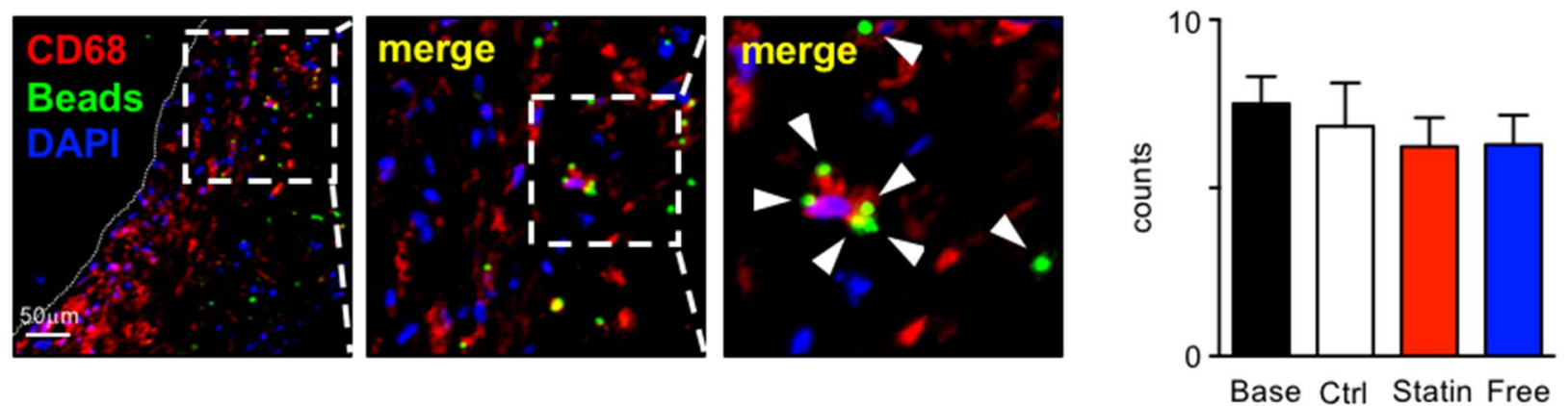

lowering or by so-called pleiotropic effects. Phenotypically, in the two APOE*3-Leiden.CETP mouse models, with and without partial body irradiation, both drug-induced and

diet-mediated normalization of cholesterol levels resulted in similar plaque regression (Figs. 1a, 2a 3a-c Supplemental Figs. 2, 4d, 5). Mechanistically, both interventions 
4Fig. 4 Serum cholesterol lowering inhibits lesional macrophage proliferation in APOE*3-Leiden.CETP mice. a Representative images of aortic root lesions stained for CD68, BrdU on the left, and quantification on the right, and $\mathbf{b}$ representative flow cytometry dot plots (left), and quantification (right) of proliferating aortic $\mathrm{M} \phi$ expressing $\mathrm{Ki} 67^{+}$and apoptotic aortic M $\phi$ expressing active Caspase $3\left(\mathrm{Casp} 3^{+}\right)$. c, d Representative images of aortic root lesions stained for CD68, TUNEL $\mathbf{c}$, and YG-fluorescent beads $\mathbf{d}$ on the left, and quantification on the right. Results are presented as mean \pm SEM, $*, \$ p<0.05$ denote statistically significant difference between the Ctrl and Statin $(*)$ or Free (§) groups, $n=8$ per group for histology and $n=6$ per group for flow cytometry readouts, one-way ANOVA suppressed local macrophage proliferation (Fig. 4a and Supplemental Figs. 2, 4d, 5 and 6a), suggesting that the cholesterol lowering effects matter. In addition, we quantified atorvastatin drug levels by mass spectrometry in livers, plasma and atherosclerotic aortas of APOE*3Leiden.CETP mice fed LCD $+0.01 \%$ atorvastatin. We detected high levels of atorvastatin in livers $(59 \mathrm{ng} / \mathrm{g})$ and lower levels in plasma $(1.9 \mathrm{ng} / \mathrm{ml})$, but virtually none in aortic tissues, arguing against atorvastatin affecting plaque macrophages directly when administered orally (Supplemental Fig. 8a, b). a
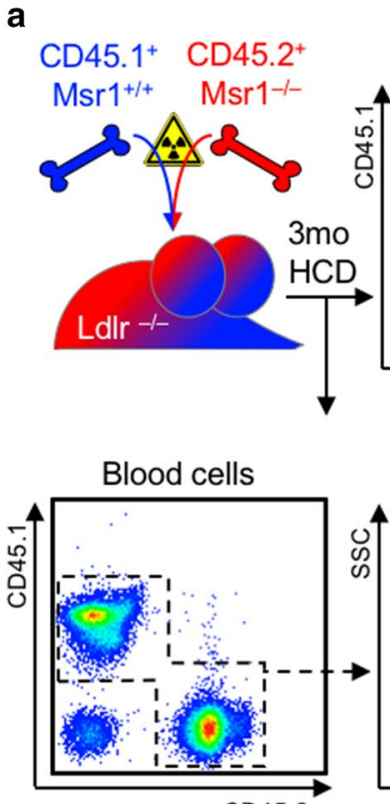

CD45.2

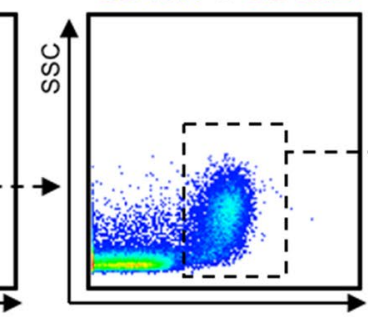

$\mathrm{CD} 11 \mathrm{~b}$
Aortic cells

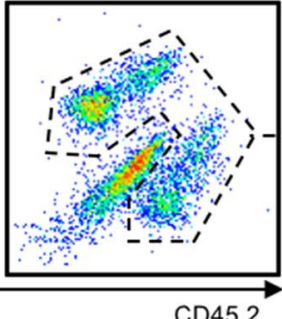

Blood leukocytes CD45.1 \& CD45.2
Aortic leukocytes

\section{CD45.1 \& CD45.2}

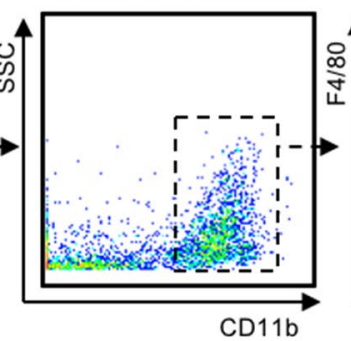

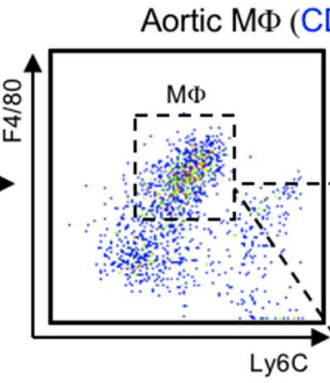

Aortic МФ (CD45.1 \& CD45.2)
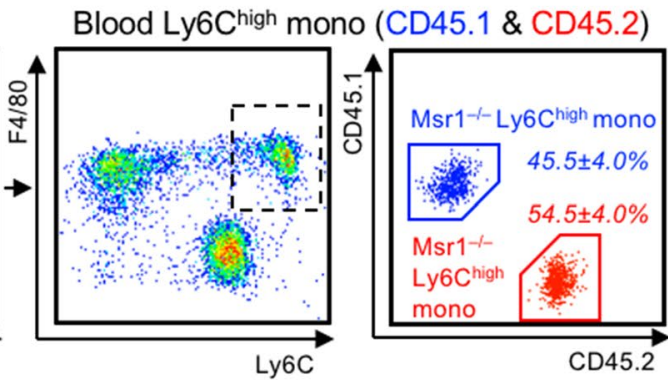

$\mathrm{CD} 45.2$

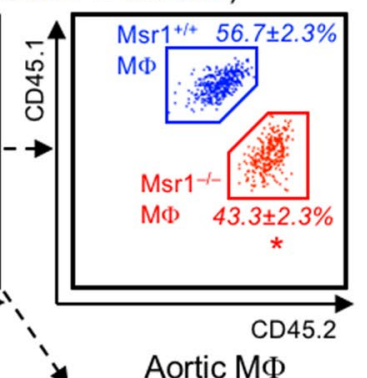

Aortic MФ

CD45.1 \& CD45.2

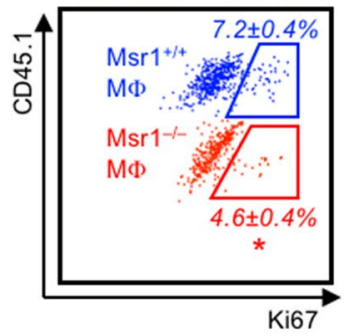

b

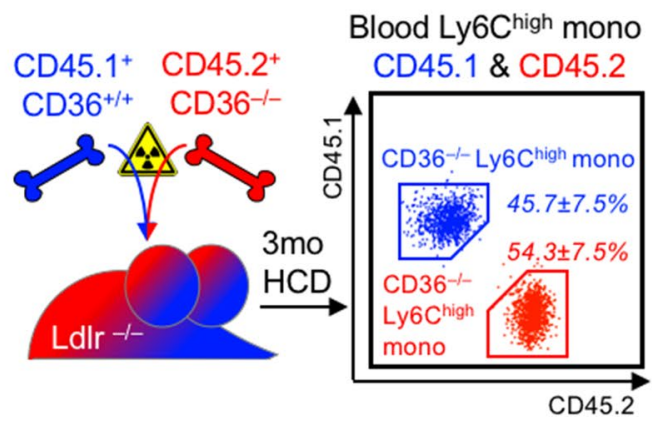

Aortic MФ

CD45.1 \& CD45.2

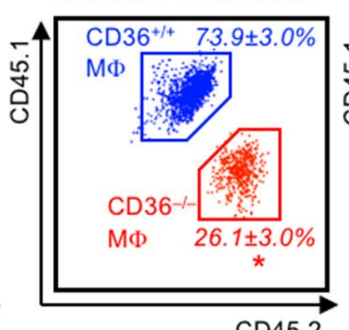

$\mathrm{CD} 45.2$

Aortic MФ

CD45.1 \& CD45.2

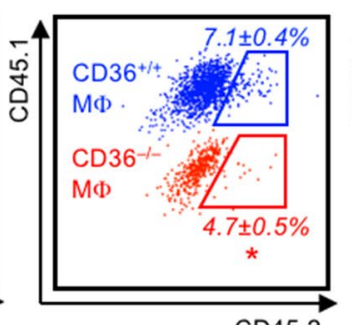

CD45.2

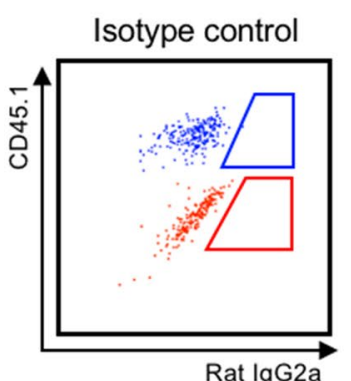

Fig. 5 Cholesterol-rich modified LDL-uptake-mediating scavenger receptors Msr1 and CD36 directly propagate MF proliferation in plaques. a $\mathrm{Ldlr}^{-/-}$mice were irradiated and reconstituted with a 1:1 mixture of CD45.1 $\mathrm{Msr}^{+/+}$and CD45.2 $\mathrm{Msr}^{-/-}$bone marrow cells for 6 weeks before starting a high-cholesterol diet (HCD) for 3 months. $\mathrm{Msr}^{+/+}$and $\mathrm{Msr}^{-/-}$Ly6 $\mathrm{C}^{\text {high }}$ monocytes (mono) in blood and macrophages $(\mathrm{M} \phi)$ in enzymatically digested atherosclerotic aortas were distinguished based on exclusive CD45.1 and CD45.2 expression, as depicted in the representative dot plots and gating strategies. The chimerism of $\mathrm{Msr} 1^{+/+}$and $\mathrm{Msr}^{-/-}$within blood Ly6C $\mathrm{C}^{\text {high }}$ monocytes and aortic M $\phi$ was quantified based on CD45.1 and CD45.2 staining, and the fraction of proliferating cells was assessed based on intracellular Ki67 staining in $\mathrm{Msr}^{+/+}$and $\mathrm{Msr}^{-/-} \mathrm{MF}$. Results are presented as mean percent \pm SEM cell chimerism and Ki $67^{+}$fraction of the respective population, $n=4$ per group, ${ }^{*} p<0.05$ denotes statistically significant differences between $\mathrm{Msr}^{+/+}$and $\mathrm{Msr}^{-/-}$Ly6C ${ }^{\text {high }}$ monocyte or M $\phi$ population, Mann-Whitney test. b Mixed CD45.1 CD36 $6^{+/+}$and $\mathrm{CD} 45.2 \mathrm{CD}^{-1-} 6^{- \text {irradiation bone mar- }}$ row chimeras were generated in $\mathrm{Ldlr}^{-1-}$ mice and analyzed in analogy to $\mathrm{Msr}^{+/+} / \mathrm{Msr}^{-/-}$chimeras, as described above. Results are presented as mean percent \pm SEM cell chimerism and $\mathrm{Ki} 67^{+}$fraction of the respective population, $n=4$ per group, $* p<0.05$ denotes statistically significant differences between $\mathrm{CD} 36^{+/+}$and $\mathrm{CD} 36^{-/-}$Ly6C ${ }^{\text {high }}$ monocyte or $\mathrm{M} \phi$ population, Mann-Whitney test 
a
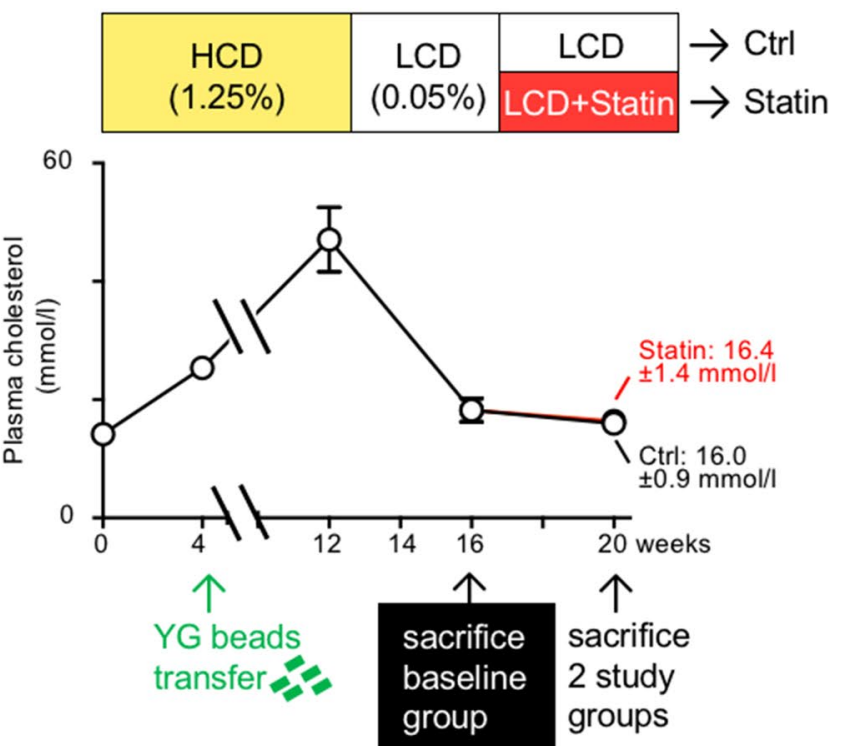

C

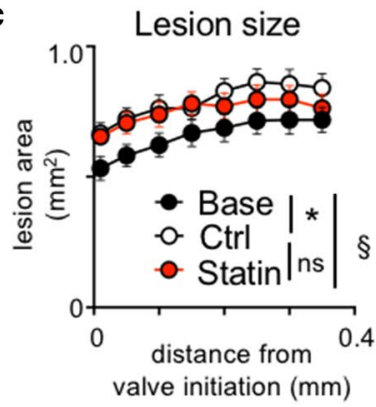

e

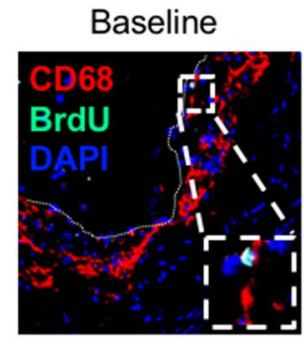

f

Baseline
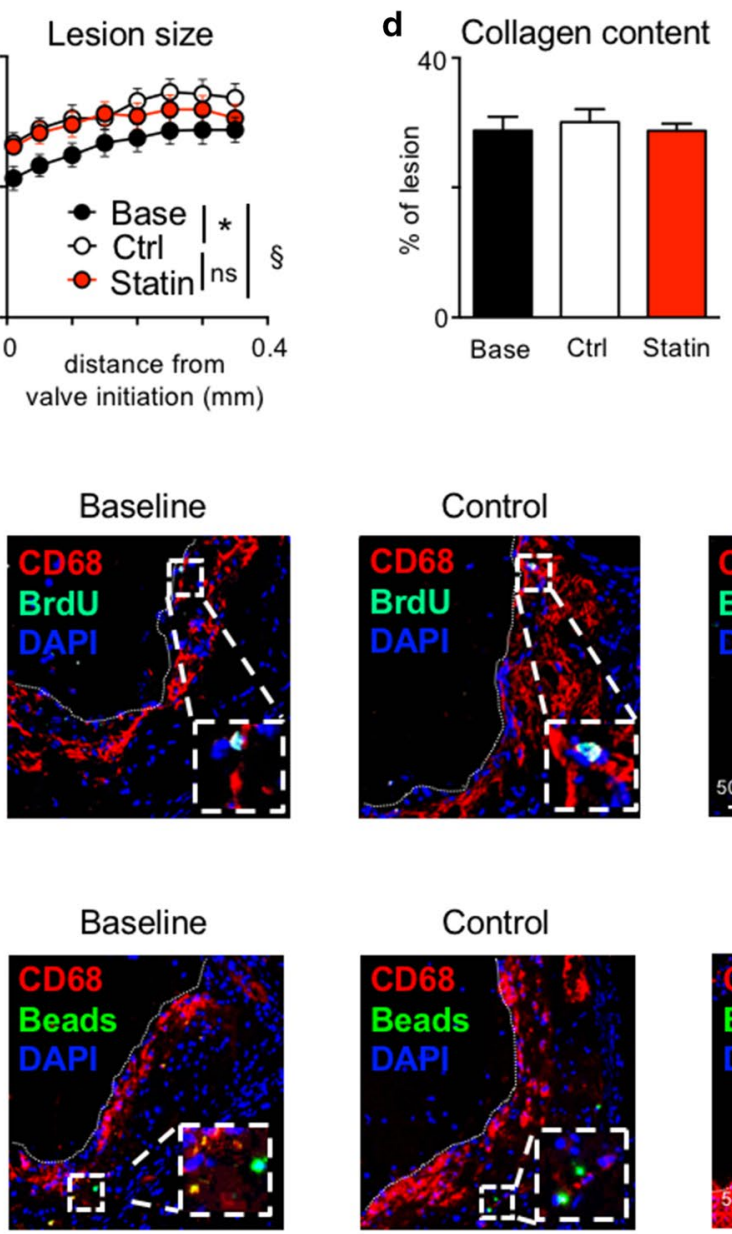

Control

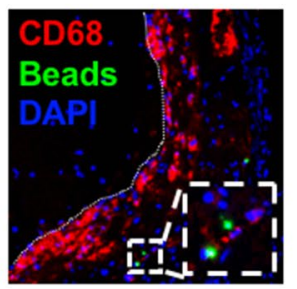

b
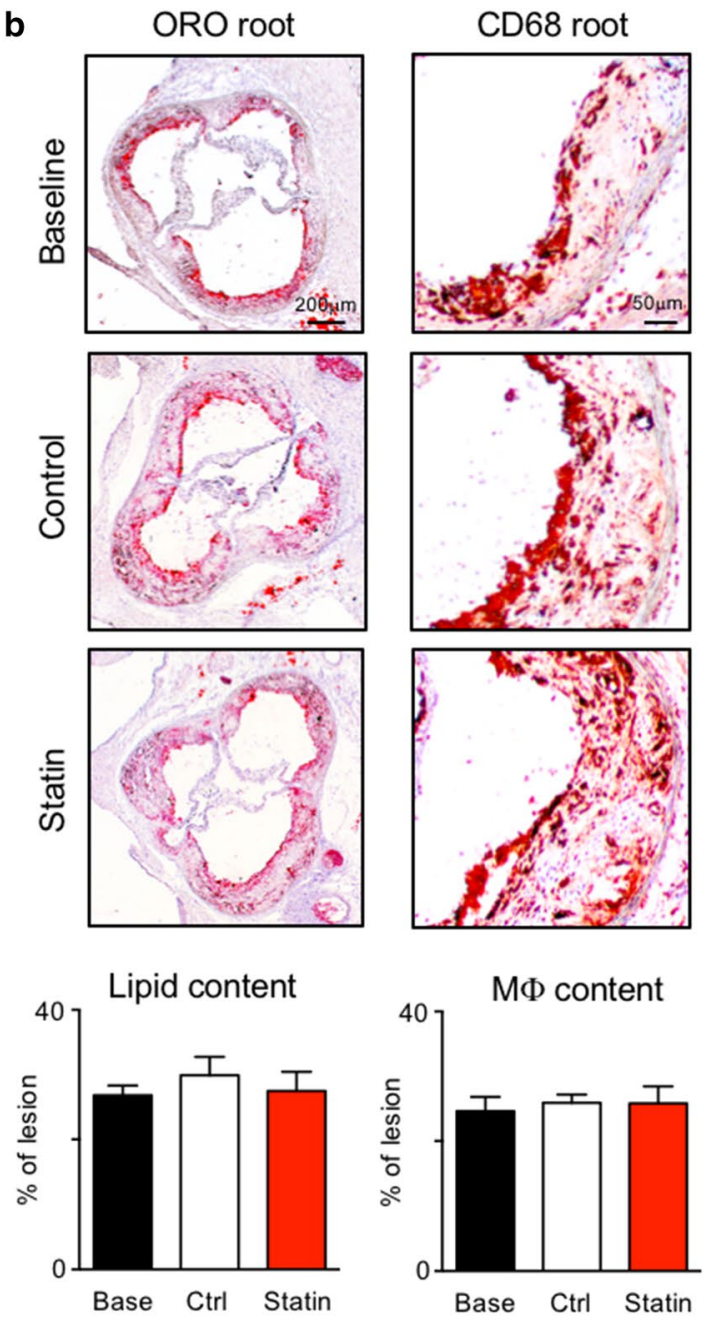

Statin

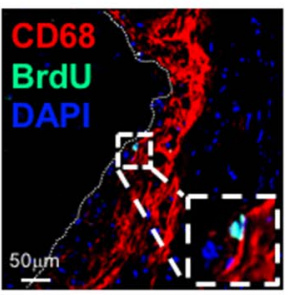

Statin

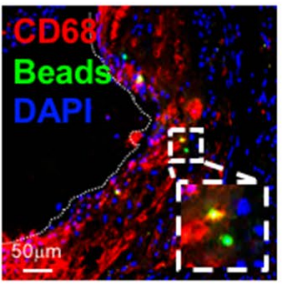

$\%$ prolif. $M \Phi$

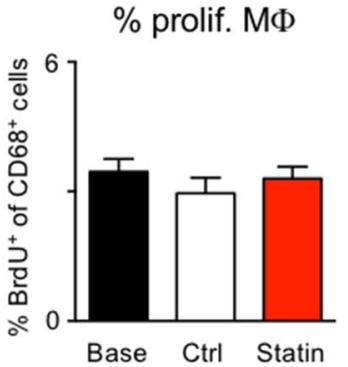

Beads per lesion

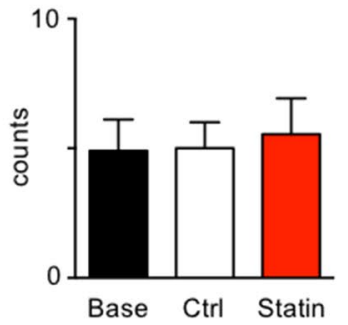


४Fig. 6 Atorvastatin fails to induce plaque regression without cholesterol lowering in $\mathrm{Apoe}^{-/-}$mice. a Study design and diet scheme similar to Fig. 1a with $\mathrm{Apoe}^{-/-}$mice fed a high-cholesterol diet $(1.25 \%$ cholesterol) for 12 weeks, followed by 4 weeks of low-cholesterol diet (LCD, $0.05 \%$ cholesterol) (Baseline, Base), and 4 weeks of continued LCD (Control, Ctrl) versus LCD supplemented with $0.01 \%$ (w/w) atorvastatin (Statin). b-d Lesion size was measured on 8 aortic root sections at $50-\mu \mathrm{m}$ intervals starting from valve initiation $\mathbf{c}$, and plaque composition was analyzed by quantifying the percent $\mathrm{ORO}^{+}, \mathrm{CD}^{+} 8^{+}$ and collagen ${ }^{+}$areas within lesions (d). Representative images of aortic root sections are show in (b) and Supplemental Fig. 8. Data are presented as mean \pm SEM. ${ }^{*}, \S p<0.05$ denote statistically significant differences between the Ctrl and Base $\left(^{*}\right)$ or Statin (§) groups, Base group $n=7$, Ctrl and Statin groups $n=8$ each, one-way ANOVA. e, f Quantification of $\mathrm{M} \phi$ proliferation based on BrdU incorporation $\mathbf{e}$ and of $\mathrm{M} \phi$ egress based on bead retention $\mathbf{f}$ in aortic root lesions. Results are presented as mean \pm SEM on the right, Base group $n=7$, Ctrl and Statin groups $n=8$ each, one-way ANOVA. Representative images are shown on the left

Given that oral atorvastatin reduces the number of circulating ApoB-lipoprotein particles and their cholesterol contents (Fig. 1a, c, d), we asked instead, whether the uptake of cholesterol-rich modified LDL into plaque macrophages will influence proliferation, directly. To this end, we generated mixed-bone marrow chimeras in irradiated $\mathrm{Ldlr}^{-1-}$ mice reconstituted with a mixture of macrophage scavenger receptor 1 (Msr1) deficient and competent bone marrow (Fig. 5a). $\mathrm{Msr}^{+/+}$bone marrow cells were isolated from CD45.1 C57B1/6 mice to distinguish wild-type from knockout cells in the reconstituted recipients according to exclusive CD45.1 or CD45.2 expression. In analogy, we generated mixed Ldlr-/- bone marrow chimeras with CD45.1 $\mathrm{CD}^{+/++}$and CD45.2 CD $36^{-/-}$cells (Fig. 5b). Msr1 preferentially mediates the uptake of LDL modified by acetylation into macrophages, whereas oxidized LDL is preferentially engulfed via CD36 (Supplemental Fig. 9a). Following 3 months of HCD feeding, atherosclerosis developed (Supplemental Fig. 9b, c), and blood and aortas were analyzed by flow cytometry for CD45.1/CD45.2 chimerism and differences in the expression of proliferation marker Ki67 in CD45.1 $\mathrm{Msr}^{+/+}$versus CD45.2 $\mathrm{Msr}^{-/-}$macrophages, and in $\mathrm{CD} 45.1 \mathrm{CD}^{+/+}$versus $\mathrm{CD} 45.2 \mathrm{CD}^{+/-} 6^{--}$macrophages, respectively. Proliferation of Msr1- or CD36-deficient macrophages was reduced by about $1 / 3$ compared to wild-type (WT) macrophages in the same plaque exposed to the same lipids and external stimuli (Fig. 5a, b). The proliferative advantage of WT macrophages over knockout macrophages may have contributed to the shift in chimerism towards CD45.1 WT macrophages observed in atherosclerotic aortas.

Finally, we treated $\mathrm{Apoe}^{-/-}$mice with established atherosclerosis in analogy to the feeding study in APOE*3Leiden.CETP mice comparing LCD with and without supplementation of $0.01 \%$ atorvastatin (Fig. 6a). As previously reported $[6,34,35,39]$, oral atorvastatin at this dose range did not reduce systemic cholesterol levels, weight gain or monocytosis in Apoe ${ }^{-/-}$mice (Fig. 6a; Supplemental Fig. 8c, d). Consequently, plaques continued to grow instead of regressing under atorvastatin treatment (Fig. 6b-d; Supplemental Fig. 8e). In the absence of a cholesterol lowering effect in $\mathrm{Apoe}^{-/-}$mice, atorvastatin did not alter macrophage accumulation, proliferation, death and retention in atherosclerotic lesions (Fig. 6e, f; Supplemental Fig. 8f, g). Taken together, these data suggest that atorvastatin inhibits plaque macrophage proliferation indirectly via lowering of cholesterol and ApoB-lipoprotein levels.

\section{Plaque macrophage proliferation in humans correlates with serum LDL-cholesterol and plaque lipid content}

Next, we asked whether the link between cholesterol levels and macrophage proliferation in atherosclerotic lesions also applied to human plaques. To this end, we collected blood and plaque tissue samples from 23 patients undergoing carotid endarterectomy (Supplemental Table 1). All but three patients were taking statins in line with guideline recommendations. Still, serum LDL-cholesterol levels ranged from $32 \mathrm{mg} / \mathrm{dl}$ to $161 \mathrm{mg} / \mathrm{dl}$. Of note, atorvastatin plasma levels in patients corresponded to those measured in our APOE*3-Leiden.CETP mouse model, and, unlike the liver, atorvastatin was hardly detectable in human plaque tissues (Supplemental Fig. 8b, Supplemental Table 2). Macrophage proliferation was assessed by co-staining DAPI, anti-CD68 and anti-Ki67 (Fig. 7b). In adjacent slides, plaque lipid content was determined based on Oil-red O staining (Fig. 7a). Macrophage proliferation correlated positively with both serum LDL-cholesterol levels $(r=0.69)$ and plaque lipid contents $(r=0.63)$ (Fig. 7c).

\section{Discussion}

We set out to investigate the cellular processes that determine plaque regression during statin treatment. Plaque progression depends on macrophage accumulation, and, conversely, macrophages vanish during plaque regression $[16,60]$. Multiple processes determine macrophage accumulation in the plaque, including monocyte recruitment and differentiation, macrophage proliferation, death and egress [32]. Macrophages of prenatal origin reside in the adventitia and self-sustain through proliferation into adulthood largely independent of monocyte recruitment [15, 65]. Whether these embryonically derived macrophages in the adventitia directly contribute to macrophages accumulating in intimal lesions is unknown. A small population of intima resident macrophages shows limited proliferation capacity and is marginalized during plaque progression [68] as monocytes infiltrate giving rise to proliferating macrophages [49]. 
Fig. 7 LDL-cholesterol correlates with local macrophage sclerotic plaques. a Representative image of a carotid artery section with plaque, where lipids are stained with Oil-red O. b Representative images of CD68 (Mф, KI67 (proliferation), Hoechst (nuclei) costaining in the plaque. Arrows mark triple-positive, proliferating $\mathrm{M \phi} \mathbf{c}$ Correlation of plaque $\mathrm{M} \phi$ proliferation rate with serum LDL-cholesterol levels and plaque lipid content $\left(\mathrm{ORO}^{+}\right.$ area), respectively. Each point represents an individual patient $(n=20), r$ : Pearson's correlation coefficient, $p<0.05$ denotes significant correlation proliferation in human athero-

a b

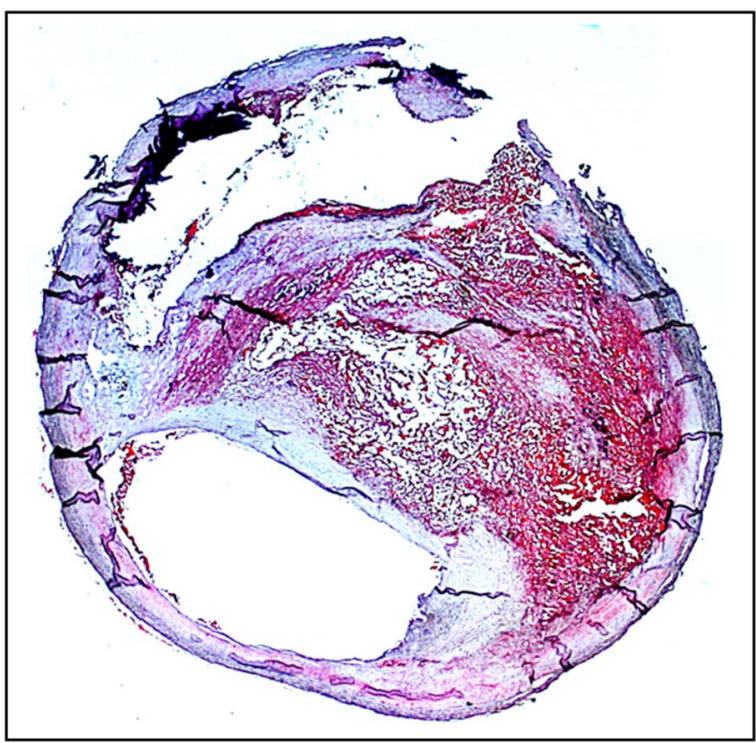

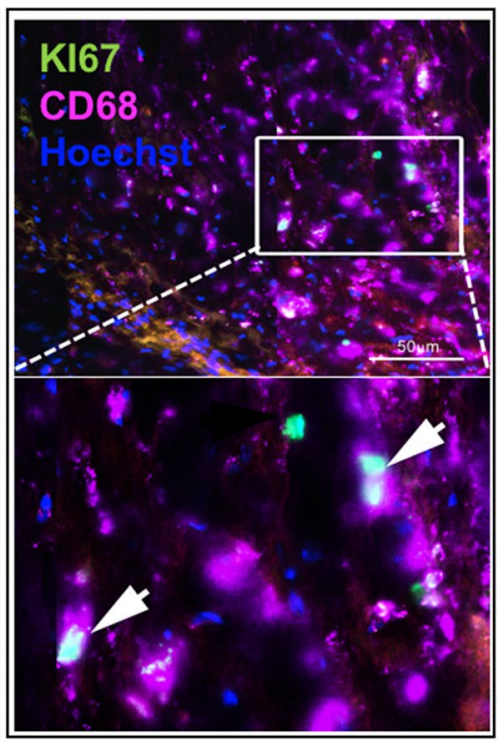

Pearson's $r=0.63 ; p<0.05$
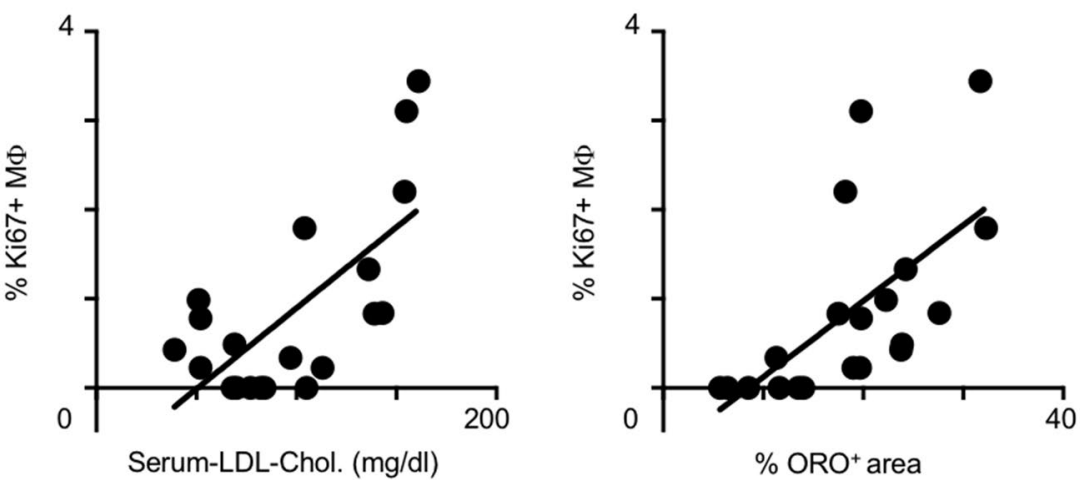

Previous experimental studies in atherosclerotic mice showed that both macrophage accumulation and plaque growth are limited by statin treatment. Most of these studies were conducted in $\mathrm{Apoe}^{-/-}$and $\mathrm{Ldlr}^{-/-}$mice, which do not respond to statins with a significant reduction in serum LDL-cholesterol at clinically relevant doses [6, 10, 34, 35]. This was interpreted as a proof of pleiotropic drug effects. Protective mechanisms proposed included suppression of endothelial cell adhesion molecule expression, stimulation of endothelial nitric oxide synthase and inhibition of leukocyte integrin LFA1 [2, 40, 66]. As a result of these molecular effects, monocyte recruitment to atherosclerotic lesions would be reduced, a finding we observed in our study as well. It was thought that impaired monocyte infiltration limited macrophage accumulation in plaques following statin treatment, but this has not been tested. Our model of irradiating mice with established atherosclerosis and aortic shielding followed by $\mathrm{GFP}^{+}$bone marrow transplantation, allowed us to quantify the contribution of blood monocytes to the plaque macrophage pool with and without plaque progression, only $11 \%$ of macrophages derived from newly recruited monocytes. These numbers matched our previous estimate of $13 \%$ monocyte contribution to the macrophage pool, based on parabiosis and BrdU incorporation in Apoe ${ }^{-/-}$mice [49]. Thus, inhibition of monocyte recruitment, as observed with atorvastatin, could not fully explain the large reduction in macrophage numbers by $40-50 \%$ in atherosclerotic lesions following atorvastatin treatment.

We, therefore, investigated cellular processes localized within the plaque, i.e. macrophage proliferation, death or egress. Indeed, macrophage proliferation, as determined by intracellular Ki67 staining and BrdU incorporation, was reduced by almost $50 \%$ by atorvastatin treatment. A previous study reported that lipophilic simvastatin, incorporated into a synthetic HDL particle, invaded the plaque, locally inhibiting macrophage proliferation and decreasing macrophage numbers in Apoe $^{-/-}$mice, without affecting serum cholesterol levels [14, 59]. Multiple in vitro studies also documented direct anti-inflammatory and anti-proliferative cholesterol lowering. We calculated that during 4 weeks of 
effects of statins on macrophages in culture [5, 52, 62]. When we treated APOE*3-Leiden.CETP mice with oral atorvastatin, achieving plasma drug levels comparable to those in patients treated with $40-80 \mathrm{mg}$ atorvastatin per day, the drug was not detected in atherosclerotic arteries, unlike the nanoparticle approach. In line, atorvastatin was also hardly detectable in human carotid artery plaques. The absence of drug accumulation in plaques does not formally exclude the possibility of local pharmacological effects. However, two additional findings in our study argue against relevant direct pleiotropic statin effects on macrophage proliferation within the plaque. First, diet induced plasma cholesterol lowering, alone, to levels achieved with oral atorvastatin treatment in APOE*3-Leiden.CETP mice yielded similar results with regard to plaque regression, dampening of systemic and local inflammation, and inhibition of macrophage proliferation in the plaque. Second, when we treated atherosclerotic $\mathrm{Apoe}^{-/}$mice with oral atorvastatin, plasma cholesterol levels remained elevated, and macrophage counts and proliferation rates were unaffected. This finding is in accordance with a previous observation we made when inhibition of monocyte production, lesion infiltration and differentiation in Apoe ${ }^{-/-}$mice with established atherosclerosis failed to slow plaque progression and accumulation of macrophages, which continued to proliferate in situ [29]. When we deleted modified lipoprotein uptake-mediating scavenger receptors (Msr1 or CD36) in macrophages accumulating in murine atherosclerotic aortas next to scavenger receptor expressing macrophages, their proliferation was relatively suppressed. These data support our hypothesis that the uptake of cholesterol-rich modified lipoproteins stimulates macrophage proliferation in atherosclerotic lesions, directly, but the intracellular signaling pathways remain to be determined. Notably, intracellular cholesterol was also found to stimulate hematopoietic stem and progenitor cell proliferation in atherosclerotic mice [33]. Oxidized LDL provokes colonystimulating factor 1 (Csf1) secretion by endothelial cells, for example [44]. A recent study described increased macrophage survival and proliferation in the plaque in response to Csf1 production by endothelial cells and vascular smooth muscle cells, in particular [57]. This may represent an additional, indirect mechanism by which statins, via systemic reduction in cholesterol-rich LDL particle numbers, inhibit lesional macrophage proliferation.

In support of our lipid uptake hypothesis, a recent experimental study comparing gene expression profiles of lipid-rich and lipid-poor macrophages from atherosclerotic murine aortas reported (in the supplement) that proliferation marker Ki67 expression was almost doubled in lipid-rich cells [22]. According to a recent meta-analysis of leukocyte diversity in atherosclerotic mouse aortas, based on singlecell RNA sequencing, these lipid-rich foamy macrophages correspond to the Trem2 (triggering receptor expressed on myeloid cells-2) macrophage subset specialized in lipid metabolism, distinct from inflammatory, resident-like and interferon-inducible macrophage subsets [71]. These macrophage populations partially overlap with subsets identified in human plaques $[12,18]$. Ingenuity pathway analysis of a single cell dataset of aortic macrophages isolated from Apoe $^{--}$mice described enrichment of proliferative, survival and motility genes in one of four macrophage clusters, as opposed to inflammation, apoptosis and phagocytosis related genes in the other clusters [31]. While these transcriptional data substantiate the presence of proliferating macrophages in atherosclerotic aortas, they do not inform on whether all subsets of lesional macrophages are equally prone to undergo cell cycling in situ. The plasticity of lesional macrophages is the focus of ongoing research. Macrophage-like cells that express macrophage markers such as CD68 and Lgals3, but not leukocyte marker CD45, may arise from vascular smooth muscle cells [54], which clonally expand in the plaque [9]. They are reported to account for 16-30\% of macrophage marker-positive cells in the plaque [1,54]. In our study in APOE*3-Leiden.CETP mice, lowering of cholesterol and atherogenic ApoB-lipoprotein levels reduced proliferation of CD68 expressing cells in general in the plaque, and this may affect macrophages of both leukocyte and vascular smooth muscle cell origin.

The relevance of macrophage egress for plaque regression is controversially debated with several papers arguing against egress [28, 29, 42, 67] and a number of papers that argue for egress $[16,30,55]$. The discrepancies may arise from differences in the models used to induce normolipidemia and timing related to bead transfer. In our models of drug- and diet-induced cholesterol lowering in APOE*3Leiden.CETP mice, and of statin treatment of $\mathrm{Apoe}^{-/-}$mice over 4 weeks, we found no indication for significant macrophage egress from established plaques, in line with the aforementioned papers.

Translating our experimental findings to humans, we showed that the levels of serum cholesterol lowering achieved in patients undergoing carotid endarterectomy is inversely correlated with the frequency of plaque macrophage proliferation. The more lipids one detects in the plaque, the higher the proportion of proliferating macrophages in situ. Studying the effects of statins, the most widely used and potent drugs in cardiovascular secondary prevention, our work documents the importance of local macrophage proliferation for plaque progression and lipid therapy-based regression. While oral atorvastatin inhibits macrophage proliferation indirectly via systemic cholesterol and ApoB-lipoprotein reductions, direct targeting of macrophage proliferation may emerge as a potent add-on therapy to support plaque regression. Anti-proliferative drugs such as paclitaxel and methotrexate or mTOR-inhibitor rapamycin, incorporated into a lipid nanoparticle LDE resembling 
low-density lipoproteins or biomimetic nanoparticles, induced plaque regression and loss of plaque macrophages in murine and rabbit models of atherosclerosis with limited systemic toxicity $[4,7,11]$. More recently, LDE-paclitaxel was injected into eight patients with aortic atherosclerosis six times every 3 weeks, and computer tomography images of the atherosclerotic aortas were compared before and after 1-2 months of treatment with those obtained in untreated patients. Remarkably, half of the treated patients showed a reduction in plaque size without significant changes to their blood cell counts, while at the same time all the nine untreated patients showed mild disease progression [56]. A number of clinical trials on treating inflammation in atherosclerosis have recently been published or are ongoing. While colchicine and canakinumab appear to protect from atherosclerotic complications post myocardial infarction [37, 38 , $47,48,61]$, low-dose methotrexate failed to do so in the latest CIRT trial [46]. Of note, at low doses and with folic acid supplementation, methotrexate does not interfere with DNA synthesis, but it may suppress inflammation via the release of adenosine [8], although no signs of modulating inflammation were seen in the CIRT trial. Whether interleukin- $1 \beta$ blockade and colchicine treatment influence plaque macrophage proliferation remains to be determined. We are finally entering an era where our conceptual understanding of the role of inflammation in atherosclerosis derived from numerous preclinical studies translates into clinics. Our study fits into this picture by identifying macrophage proliferation as a relevant and modifiable determinant of inflammatory cell accumulation in atherosclerotic lesions to be targeted therapeutically in support of plaque regression.

Acknowledgements We thank Sandrine Villoin and Guillaume Hochart from ImaBiotech for excellent help and work with mass spectrometry, and the microscopy facility SCI-MED (Super-Resolution Confocal/Multiphoton Imaging for Multiparametric Experimental Designs) at the Institute for Experimental Cardiovascular Medicine for providing access to and support for the confocal microscope.

Author contributions $\mathrm{CH}$ and JK contributed equally by acquiring, analyzing and interpreting the data, and assisting in writing the manuscript; KK, CAE, AJ, JZ, CS, SR, DS, BD, NH, TD, RK, TB, and BHN acquired and analyzed data; FW, PS, TH, DW, CzM, JM, PK, EJP, HP, FKS, CSR, CB, and AZ helped to interpreted the data and to edit the manuscript; IH conceived and supervised the project, analyzed and interpreted the data, wrote the manuscript, and acquired funding.

Funding Open Access funding enabled and organized by Projekt DEAL. This work was supported by the German Research Foundation to I.H. (HI1573/2) and the Collaborative Research Centre SFB1425 (Grant 422681845), and the TNO research program "Preventive Health Technologies".

Data availability Data are available from the corresponding author upon reasonable request.

\section{Compliance with ethical standards}

Conflict of interest The authors declare no conflict of interest.

Ethical approval All procedures involving animals were approved by the Animal Care Committee of the University of Freiburg and the Regional Council. All experiments involving human samples were approved by the Institutional Review Board of the University Hospital of Freiburg. Written informed consent was obtained from participants prior to inclusion in the study.

Open Access This article is licensed under a Creative Commons Attribution 4.0 International License, which permits use, sharing, adaptation, distribution and reproduction in any medium or format, as long as you give appropriate credit to the original author(s) and the source, provide a link to the Creative Commons licence, and indicate if changes were made. The images or other third party material in this article are included in the article's Creative Commons licence, unless indicated otherwise in a credit line to the material. If material is not included in the article's Creative Commons licence and your intended use is not permitted by statutory regulation or exceeds the permitted use, you will need to obtain permission directly from the copyright holder. To view a copy of this licence, visit http://creativecommons.org/licenses/by/4.0/.

\section{References}

1. Albarrán-Juárez J, Kaur H, Grimm M, Offermanns S, Wettschureck N (2016) Lineage tracing of cells involved in atherosclerosis. Atherosclerosis 251:445-453. https://doi.org/10.1016/j. atherosclerosis.2016.06.012

2. Babelova A, Sedding DG, Brandes RP (2013) Anti-atherosclerotic mechanisms of statin therapy. Curr Opin Pharmacol 13:260-264. https://doi.org/10.1016/j.coph.2013.01.004

3. Bea F, Blessing E, Bennett B, Levitz M, Wallace EP, Rosenfeld ME (2002) Simvastatin promotes atherosclerotic plaque stability in apoE-deficient mice independently of lipid lowering. Arterioscler Thromb Vasc Biol 22:1832-1837. https://doi. org/10.1161/01.ATV.0000036081.01231.16

4. Boada C, Zinger A, Tsao C, Zhao P, Martinez JO, Hartman K, Naoi T, Sukhoveshin R, Sushnitha M, Molinaro R, Trachtenberg B, Cooke JP, Tasciotti E (2020) Rapamycin-loaded biomimetic nanoparticles reverse vascular inflammation. Circ Res 126:25-37. https://doi.org/10.1161/CIRCRESAHA.119.315185

5. Bonetti PO, Lerman LO, Napoli C, Lerman A (2003) Statin effects beyond lipid lowering - are they clinically relevant. Eur Heart J 24:225-248. https://doi.org/10.1016/S0195-668X0200419-0

6. Bot I, Jukema JW, Lankhuizen IM, van Berkel TJ, Biessen EA (2011) Atorvastatin inhibits plaque development and adventitial neovascularization in ApoE deficient mice independent of plasma cholesterol levels. Atherosclerosis 214:295-300. https:// doi.org/10.1016/j.atherosclerosis.2010.11.008

7. Bulgarelli A, Leite ACJ, Dias AA, Maranhao RC (2013) Antiatherogenic effects of methotrexate carried by a lipid nanoemulsion that binds to LDL receptors in cholesterol-fed rabbits. Cardiovasc Drugs Ther 27:531-539. https://doi.org/10.1007/s1055 7-013-6488-3

8. Chan ES, Cronstein BN (2010) Methotrexate-how does it really work. Nat Rev Rheumatol 6:175-178. https://doi.org/10.1038/ nrrheum.2010.5 
9. Chappell J, Harman JL, Narasimhan VM, Yu H, Foote K, Simons BD, Bennett MR, Jørgensen HF (2016) Extensive proliferation of a subset of differentiated, yet plastic, medial vascular smooth muscle cells contributes to neointimal formation in mouse injury and atherosclerosis models. Circ Res 119:1313-1323. https://doi. org/10.1161/CIRCRESAHA.116.309799

10. Chen Z, Fukutomi T, Zago AC, Ehlers R, Detmers PA, Wright SD, Rogers C, Simon DI (2002) Simvastatin reduces neointimal thickening in low-density lipoprotein receptor-deficient mice after experimental angioplasty without changing plasma lipids. Circulation 106:20-23. https://doi.org/10.1161/01.CIR.0000022843 .76104 .01

11. Lima D, A, Hua N, C Maranhao R, A Hamilton J, (2017) Evaluation of atherosclerotic lesions in cholesterol-fed mice during treatment with paclitaxel in lipid nanoparticles: a magnetic resonance imaging study. J Biomed Res 31:116-121. https://doi.org/10.7555/ JBR.31.20160123

12. Depuydt MA, Prange KH, Slenders L, Örd T, Elbersen D, Boltjes A, de Jager SC, Asselbergs FW, de Borst GJ, Aavik E, Lönnberg T, Lutgens E, Glass CK, den Ruijter HM, Kaikkonen MU, Bot I, Slütter B, van der Laan SW, Yla-Herttuala S, Mokry M, Kuiper J, de Winther MP, Pasterkamp G (2020) Microanatomy of the human atherosclerotic plaque by single-cell transcriptomics. Circ Res. https://doi.org/10.1161/CIRCRESAHA.120.316770

13. Distel E, Barrett TJ, Chung K, Girgis NM, Parathath S, Essau CC, Murphy AJ, Moore KJ, Fisher EA (2014) miR33 inhibition overcomes deleterious effects of diabetes mellitus on atherosclerosis plaque regression in mice. Circ Res 115:759-769. https:// doi.org/10.1161/CIRCRESAHA.115.304164

14. Duivenvoorden R, Tang J, Cormode DP, Mieszawska AJ, Izquierdo-Garcia D, Ozcan C, Otten MJ, Zaidi N, Lobatto ME, van Rijs SM, Priem B, Kuan EL, Martel C, Hewing B, Sager H, Nahrendorf M, Randolph GJ, Stroes ES, Fuster V, Fisher EA, Fayad ZA, Mulder WJ (2014) A statin-loaded reconstituted high-density lipoprotein nanoparticle inhibits atherosclerotic plaque inflammation. Nat Commun 5:3065. https://doi.org/10.1038/ncomms4065

15. Ensan S, Li A, Besla R, Degousee N, Cosme J, Roufaiel M, Shikatani EA, El-Maklizi M, Williams JW, Robins L, Li C, Lewis B, Yun TJ, Lee JS, Wieghofer P, Khattar R, Farrokhi K, Byrne J, Ouzounian M, Zavitz CC, Levy GA, Bauer CM, Libby P, Husain M, Swirski FK, Cheong C, Prinz M, Hilgendorf I, Randolph GJ, Epelman S, Gramolini AO, Cybulsky MI, Rubin BB, Robbins CS (2016) Self-renewing resident arterial macrophages arise from embryonic CX3CR1(+) precursors and circulating monocytes immediately after birth. Nat Immunol 17:159-168. https://doi. org/10.1038/ni.3343

16. Feig JE, Parathath S, Rong JX, Mick SL, Vengrenyuk Y, Grauer L, Young SG, Fisher EA (2011) Reversal of hyperlipidemia with a genetic switch favorably affects the content and inflammatory state of macrophages in atherosclerotic plaques. Circulation 123:989998. https://doi.org/10.1161/CIRCULATIONAHA.110.984146

17. Ference BA, Ginsberg HN, Graham I, Ray KK, Packard CJ, Bruckert E, Hegele RA, Krauss RM, Raal FJ, Schunkert H, Watts GF, Boren J, Fazio S, Horton JD, Masana L, Nicholls SJ, Nordestgaard BG, van de Sluis B, Taskinen MR, Tokgozoglu L, Landmesser U, Laufs U, Wiklund O, Stock JK, Chapman MJ, Catapano AL (2017) Low-density lipoproteins cause atherosclerotic cardiovascular disease. 1. Evidence from genetic, epidemiologic, and clinical studies. A consensus statement from the European Atherosclerosis Society Consensus Panel. Eur Heart J 38:2459-2472. https://doi.org/10.1093/eurheartj/ehx144

18. Fernandez DM, Rahman AH, Fernandez NF, Chudnovskiy A, Amir ED, Amadori L, Khan NS, Wong CK, Shamailova R, Hill CA, Wang Z, Remark R, Li JR, Pina C, Faries C, Awad AJ, Moss N, Bjorkegren JLM, Kim-Schulze S, Gnjatic S, Ma'ayan A, Mocco J, Faries P, Merad M, Giannarelli C (2019) Single-cell immune landscape of human atherosclerotic plaques. Nat Med 25:1576-1588. https://doi.org/10.1038/s41591-019-0590-4

19. Gordon D, Reidy MA, Benditt EP, Schwartz SM (1990) Cell proliferation in human coronary arteries. Proc Natl Acad Sci USA 87:4600-4604. https://doi.org/10.1073/pnas.87.12.4600

20. Grundy SM, Stone NJ, Bailey AL, Beam C, Birtcher KK, Blumenthal RS, Braun LT, de Ferranti S, Faiella-Tommasino J, Forman DE, Goldberg R, Heidenreich PA, Hlatky MA, Jones DW, Lloyd-Jones D, Lopez-Pajares N, Ndumele CE, Orringer CE, Peralta CA, Saseen JJ, Smith SCJ, Sperling L, Virani SS, Yeboah J (2018) 2018 AHA/ACC/AACVPR/AAPA/ABC/ACPM/ADA/ AGS/APhA/ASPC/NLA/PCNA Guideline on the Management of Blood Cholesterol: a report of the American college of cardiology/ American heart association task force on clinical practice guidelines. J Am Coll Cardiol 73:e285-e350. https://doi.org/10.1016/j. jacc.2018.11.003

21. Haka AS, Potteaux S, Fraser H, Randolph GJ, Maxfield FR (2012) Quantitative analysis of monocyte subpopulations in murine atherosclerotic plaques by multiphoton microscopy. PLoS ONE 7:e44823. https://doi.org/10.1371/journal.pone.0044823

22. Kim K, Shim D, Lee JS, Zaitsev K, Williams JW, Kim K-W, Jang M-Y, Seok Jang H, Yun TJ, Lee SH, Yoon WK, Prat A, Seidah NG, Choi J, Lee S-P, Yoon S-H, Nam JW, Seong JK, Oh GT, Randolph GJ, Artyomov MN, Cheong C, Choi J-H (2018) Transcriptome analysis reveals nonfoamy rather than foamy plaque macrophages are proinflammatory in atherosclerotic murine models. Circ Res 123:1127-1142. https://doi.org/10.1161/CIRCRESAHA .118 .312804

23. Kinlay S (2007) Low-density lipoprotein-dependent and -independent effects of cholesterol-lowering therapies on C-reactive protein: a meta-analysis. J Am Coll Cardiol 49:2003-2009. https ://doi.org/10.1016/j.jacc.2007.01.083

24. Koskinas KC, Ughi GJ, Windecker S, Tearney GJ, Raber L (2016) Intracoronary imaging of coronary atherosclerosis: validation for diagnosis, prognosis and treatment. Eur Heart J 37:524-535. https ://doi.org/10.1093/eurheartj/ehv642

25. Kühnast S, Fiocco M, van der Hoorn JW, Princen HM, Jukema JW (2015) Innovative pharmaceutical interventions in cardiovascular disease: Focusing on the contribution of non-HDL-C/ LDL-C-lowering versus HDL-C-raising: A systematic review and meta-analysis of relevant preclinical studies and clinical trials. Eur J Pharmacol 763:48-63. https://doi.org/10.1016/j.ejpha r.2015.03.089

26. Kuhnast $S$, van der Tuin SJ, van der Hoorn JW, van Klinken JB, Simic B, Pieterman E, Havekes LM, Landmesser U, Luscher TF, Willems van Dijk K, Rensen PC, Jukema JW, Princen HM (2015) Anacetrapib reduces progression of atherosclerosis, mainly by reducing non-HDL-cholesterol, improves lesion stability and adds to the beneficial effects of atorvastatin. Eur Heart J 36:39-48. https://doi.org/10.1093/eurheartj/ehu319

27. Lamon-Fava S, Diffenderfer MR, Barrett PH, Buchsbaum A, Matthan NR, Lichtenstein AH, Dolnikowski GG, Horvath K, Asztalos BF, Zago V, Schaefer EJ (2007) Effects of different doses of atorvastatin on human apolipoprotein B-100, B-48, and A-I metabolism. J Lipid Res 48:1746-1753. https://doi.org/10.1194/ jlr.M700067-JLR200

28. Li W, Luehmann HP, Hsiao H-M, Tanaka S, Higashikubo R, Gauthier JM, Sultan D, Lavine KJ, Brody SL, Gelman AE, Gropler RJ, Liu Y, Kreisel D (2018) Visualization of monocytic cells in regressing atherosclerotic plaques by intravital 2-photon and positron emission tomography-based imaging-brief report. Arterioscler Thromb Vasc Biol 38:1030-1036. https://doi.org/10.1161/ ATVBAHA.117.310517

29. Lindau A, Hardtner C, Hergeth SP, Blanz KD, Dufner B, Hoppe N, Anto-Michel N, Kornemann J, Zou J, Gerhardt LM, Heidt T, Willecke F, Geis S, Stachon P, Wolf D, Libby P, Swirski FK, 
Robbins CS, McPheat W, Hawley S, Braddock M, Gilsbach R, Hein L, von zur Muhlen C, Bode C, Zirlik A, Hilgendorf I (2016) Atheroprotection through SYK inhibition fails in established disease when local macrophage proliferation dominates lesion progression. Basic Res Cardiol 111:20. https://doi.org/10.1007/s0039 5-016-0535-8

30. Llodra J, Angeli V, Liu J, Trogan E, Fisher EA, Randolph GJ (2004) Emigration of monocyte-derived cells from atherosclerotic lesions characterizes regressive, but not progressive, plaques. Proc Natl Acad Sci U S A 101:11779-11784. https://doi.org/10.1073/ pnas.0403259101

31. McArdle S, Buscher K, Ghosheh Y, Pramod AB, Miller J, Winkels H, Wolf D, Ley K (2019) Migratory and dancing macrophage subsets in atherosclerotic lesions. Circ Res 125:1038-1051. https ://doi.org/10.1161/CIRCRESAHA.119.315175

32. Moore KJ, Koplev S, Fisher EA, Tabas I, Bjorkegren JLM, Doran AC, Kovacic JC (2018) Macrophage trafficking, inflammatory resolution, and genomics in atherosclerosis: JACC macrophage in CVD series (Part 2). J Am Coll Cardiol 72:2181-2197. https:// doi.org/10.1016/j.jacc.2018.08.2147

33. Murphy AJ, Akhtari M, Tolani S, Pagler T, Bijl N, Kuo CL, Wang M, Sanson M, Abramowicz S, Welch C, Bochem AE, Kuivenhoven JA, Yvan-Charvet L, Tall AR (2011) ApoE regulates hematopoietic stem cell proliferation, monocytosis, and monocyte accumulation in atherosclerotic lesions in mice. J Clin Invest 121:4138-4149. https://doi.org/10.1172/JCI57559

34. Nachtigal P, Jamborova G, Pospisilova N, Pospechova K, Solichova D, Zdansky P, Semecky V (2006) Atorvastatin has distinct effects on endothelial markers in different mouse models of atherosclerosis. J Pharm Pharm Sci 9:222-230

35. Nachtigal P, Pospisilova N, Jamborova G, Pospechova K, Solichova D, Andrys C, Zdansky P, Micuda S, Semecky V (2008) Atorvastatin has hypolipidemic and anti-inflammatory effects in apoE/LDL receptor-double-knockout mice. Life Sci 82:708-717. https://doi.org/10.1016/j.lfs.2008.01.006

36. Nair AB, Jacob S (2016) A simple practice guide for dose conversion between animals and human. J Basic Clin Pharm 7:27-31. https://doi.org/10.4103/0976-0105.177703

37. Nidorf SM, Eikelboom JW, Budgeon CA, Thompson PL (2013) Low-dose colchicine for secondary prevention of cardiovascular disease. J Am Coll Cardiol 61:404-410. https://doi.org/10.1016/j. jacc.2012.10.027

38. Nidorf SM, Fiolet ATL, Mosterd A, Eikelboom JW, Schut A, Opstal TSJ, The SHK, Xu XF, Ireland MA, Lenderink T, Latchem D, Hoogslag P, Jerzewski A, Nierop P, Whelan A, Hendriks R, Swart H, Schaap J, Kuijper AFM, van Hessen MWJ, Saklani P, Tan I, Thompson AG, Morton A, Judkins C, Bax WA, Dirksen M, Alings M, Hankey GJ, Budgeon CA, Tijssen JGP, Cornel JH, Thompson PL, LoDoCo2 T I (2020) Colchicine in patients with chronic coronary disease. N Engl J Med. https://doi.org/10.1056/ NEJMoa2021372

39. Nie P, Li D, Hu L, Jin S, Yu Y, Cai Z, Shao Q, Shen J, Yi J, Xiao $\mathrm{H}$, Shen L, He B (2014) Atorvastatin improves plaque stability in ApoE-knockout mice by regulating chemokines and chemokine receptors. PLoS ONE 9:e97009. https://doi.org/10.1371/journ al.pone.0097009

40. Oesterle A, Laufs U, Liao JK (2017) Pleiotropic effects of statins on the cardiovascular system. Circ Res 120:229-243. https://doi. org/10.1161/CIRCRESAHA.116.308537

41. Piepoli MF, Hoes AW, Agewall S, Albus C, Brotons C, Catapano AL, Cooney MT, Corra U, Cosyns B, Deaton C, Graham I, Hall MS, Hobbs FDR, Lochen ML, Lollgen H, Marques-Vidal P, Perk J, Prescott E, Redon J, Richter DJ, Sattar N, Smulders Y, Tiberi M, van der Worp HB, van Dis I, Verschuren WMM, Binno S (2016) 2016 European guidelines on cardiovascular disease prevention in clinical practice: the sixth joint task force of the European society of cardiology and other societies on cardiovascular disease prevention in clinical practice (constituted by representatives of 10 societies and by invited experts)developed with the special contribution of the European association for cardiovascular prevention and rehabilitation (EACPR). Eur Heart J 37:2315-2381. https:// doi.org/10.1093/eurheartj/ehw106

42. Potteaux S, Gautier EL, Hutchison SB, van Rooijen N, Rader DJ, Thomas MJ, Sorci-Thomas MG, Randolph GJ (2011) Suppressed monocyte recruitment drives macrophage removal from atherosclerotic plaques of Apoe-/- mice during disease regression. J Clin Invest 121:2025-2036. https://doi.org/10.1172/JCI43802

43. Rahman K, Vengrenyuk Y, Ramsey SA, Vila NR, Girgis NM, Liu J, Gusarova V, Gromada J, Weinstock A, Moore KJ, Loke P, Fisher EA (2017) Inflammatory Ly6Chi monocytes and their conversion to M2 macrophages drive atherosclerosis regression. J Clin Invest 127:2904-2915. https://doi.org/10.1172/JCI75005

44. Rajavashisth TB, Andalibi A, Territo MC, Berliner JA, Navab M, Fogelman AM, Lusis AJ (1990) Induction of endothelial cell expression of granulocyte and macrophage colony-stimulating factors by modified low-density lipoproteins. Nature 344:254257. https://doi.org/10.1038/344254a0

45. Rekhter MD, Gordon D (1995) Active proliferation of different cell types, including lymphocytes, in human atherosclerotic plaques. Am J Pathol 147:668-677

46. Ridker PM, Everett BM, Pradhan A, MacFadyen JG, Solomon DH, Zaharris E, Mam V, Hasan A, Rosenberg Y, Iturriaga E, Gupta M, Tsigoulis M, Verma S, Clearfield M, Libby P, Goldhaber SZ, Seagle R, Ofori C, Saklayen M, Butman S, Singh N, Le May M, Bertrand O, Johnston J, Paynter NP, Glynn RJ (2018) Low-dose methotrexate for the prevention of atherosclerotic events. N Engl J Med. https://doi.org/10.1056/NEJMoa1809798

47. Ridker PM, Everett BM, Thuren T, MacFadyen JG, Chang WH, Ballantyne C, Fonseca F, Nicolau J, Koenig W, Anker SD, Kastelein JJP, Cornel JH, Pais P, Pella D, Genest J, Cifkova R, Lorenzatti A, Forster T, Kobalava Z, Vida-Simiti L, Flather M, Shimokawa H, Ogawa H, Dellborg M, Rossi PRF, Troquay RPT, Libby P, GlynnCantos RJTG (2017) Antiinflammatory therapy with canakinumab for atherosclerotic disease. N Engl J Med 377:1119-1131. https://doi.org/10.1056/NEJMoa1707914

48. Ridker PM, Libby P, MacFadyen JG, Thuren T, Ballantyne C, Fonseca F, Koenig W, Shimokawa H, Everett BM, Glynn RJ (2018) Modulation of the interleukin-6 signalling pathway and incidence rates of atherosclerotic events and all-cause mortality: analyses from the canakinumab anti-inflammatory thrombosis outcomes study (CANTOS). Eur Heart J 39:3499-3507. https:// doi.org/10.1093/eurheartj/ehy310

49. Robbins CS, Hilgendorf I, Weber GF, Theurl I, Iwamoto Y, Figueiredo JL, Gorbatov R, Sukhova GK, Gerhardt LM, Smyth D, Zavitz CC, Shikatani EA, Parsons M, van Rooijen N, Lin HY, Husain M, Libby P, Nahrendorf M, Weissleder R, Swirski FK (2013) Local proliferation dominates lesional macrophage accumulation in atherosclerosis. Nat Med 19:1166-1172. https://doi. org/10.1038/nm.3258

50. Rosenfeld ME, Ross R (1990) Macrophage and smooth muscle cell proliferation in atherosclerotic lesions of WHHL and comparably hypercholesterolemic fat-fed rabbits. Arteriosclerosis 10:680-687. https://doi.org/10.1161/01.atv.10.5.680

51. Roth L, Rombouts M, Schrijvers DM, Martinet W, De Meyer GR (2016) Cholesterol-independent effects of atorvastatin prevent cardiovascular morbidity and mortality in a mouse model of atherosclerotic plaque rupture. Vascul Pharmacol 80:50-58. https:// doi.org/10.1016/j.vph.2016.01.007

52. Sakai M, Kobori S, Matsumura T, Biwa T, Sato Y, Takemura T, Hakamata H, Horiuchi S, Shichiri M (1997) HMG-CoA reductase inhibitors suppress macrophage growth induced by oxidized 
low density lipoprotein. Atherosclerosis 133:51-59. https://doi. org/10.1016/s0021-9150(97)00118-4

53. Schaefer EJ, McNamara JR, Tayler T, Daly JA, Gleason JL, Seman LJ, Ferrari A, Rubenstein JJ (2004) Comparisons of effects of statins (atorvastatin, fluvastatin, lovastatin, pravastatin, and simvastatin) on fasting and postprandial lipoproteins in patients with coronary heart disease versus control subjects. Am J Cardiol 93:31-39. https://doi.org/10.1016/j.amjcard.2003.09.008

54. Shankman LS, Gomez D, Cherepanova OA, Salmon M, Alencar GF, Haskins RM, Swiatlowska P, Newman AA, Greene ES, Straub AC, Isakson B, Randolph GJ, Owens GK (2015) KLF4-dependent phenotypic modulation of smooth muscle cells has a key role in atherosclerotic plaque pathogenesis. Nat Med 21:628-637. https ://doi.org/10.1038/nm.3866

55. Sharma M, Schlegel MP, Afonso MS, Brown EJ, Rahman K, Weinstock A, Sansbury B, Corr EM, van Solingen C, Koelwyn G, Shanley LC, Beckett L, Peled D, Lafaille JJ, Spite M, Loke P, Fisher EA, Moore KJ (2020) Regulatory T cells license macrophage pro-resolving functions during atherosclerosis regression. Circ Res. https://doi.org/10.1161/CIRCRESAHA.119.316461

56. Shiozaki AA, Senra T, Morikawa AT, Deus DF, Paladino-Filho AT, Pinto IM, Maranhao RC (2016) Treatment of patients with aortic atherosclerotic disease with paclitaxel-associated lipid nanoparticles. Clinics (Sao Paulo) 71:435-439. https://doi. org/10.6061/clinics/2016(08)05

57. Sinha SK, Miikeda A, Fouladian Z, Mehrabian M, Edillor C, Shih D, Zhou Z, Paul MK, Charugundla S, Davis RC, Rajavashisth TB, Lusis AJ (2020) Local M-CSF (macrophage colony-stimulating factor) expression regulates macrophage proliferation and apoptosis in atherosclerosis. Arterioscler Thromb Vasc Biol. https:// doi.org/10.1161/ATVBAHA.120.315255

58. Sniderman AD, Thanassoulis G, Glavinovic T, Navar AM, Pencina M, Catapano A, Ference BA (2019) Apolipoprotein B particles and cardiovascular disease: a narrative review. JAMA Cardiol 4:1287-1295. https://doi.org/10.1001/jamacardio .2019 .3780

59. Tang J, Lobatto ME, Hassing L, van der Staay S, van Rijs SM, Calcagno C, Braza MS, Baxter S, Fay F, Sanchez-Gaytan BL, Duivenvoorden R, Sager H, Astudillo YM, Leong W, Ramachandran S, Storm G, Perez-Medina C, Reiner T, Cormode DP, Strijkers GJ, Stroes ES, Swirski FK, Nahrendorf M, Fisher EA, Fayad ZA, Mulder WJ (2015) Inhibiting macrophage proliferation suppresses atherosclerotic plaque inflammation. Sci Adv. https://doi. org/10.1126/sciadv.1400223

60. Tang TY, Howarth SP, Miller SR, Graves MJ, Patterson AJ, U-King-Im JM, Li ZY, Walsh SR, Brown AP, Kirkpatrick PJ, Warburton EA, Hayes PD, Varty K, Boyle JR, Gaunt ME, Zalewski A, Gillard JH (2009) The ATHEROMA (Atorvastatin therapy: effects on reduction of macrophage activity) study. Evaluation using ultrasmall superparamagnetic iron oxide-enhanced magnetic resonance imaging in carotid disease. J Am Coll Cardiol 53:2039-2050. https://doi.org/10.1016/j.jacc.2009.03.018

61. Tardif JC, Kouz S, Waters DD, Bertrand OF, Diaz R, Maggioni AP, Pinto FJ, Ibrahim R, Gamra H, Kiwan GS, Berry C, LópezSendón J, Ostadal P, Koenig W, Angoulvant D, Grégoire JC, Lavoie MA, Dubé MP, Rhainds D, Provencher M, Blondeau L, Orfanos A, L'Allier PL, Guertin MC, Roubille F (2019) Efficacy and safety of low-dose colchicine after myocardial infarction. $\mathrm{N}$ Engl J Med 381:2497-2505. https://doi.org/10.1056/NEJMoa1912 388

62. Tuomisto TT, Lumivuori H, Kansanen E, Hakkinen SK, Turunen MP, van Thienen JV, Horrevoets AJ, Levonen AL, Yla-Herttuala S (2008) Simvastatin has an anti-inflammatory effect on macrophages via upregulation of an atheroprotective transcription factor, Kruppel-like factor 2. Cardiovasc Res 78:175-184. https:// doi.org/10.1093/cvr/cvn007

63. van Vlijmen BJ, van 't Hof $\mathrm{HB}, \mathrm{Mol} M J$, van der Boom $\mathrm{H}$, van der Zee A, Frants RR, Hofker MH, Havekes LM (1996) Modulation of very low density lipoprotein production and clearance contributes to age- and gender- dependent hyperlipoproteinemia in apolipoprotein E3-Leiden transgenic mice. J Clin Invest 97:1184-1192. https://doi.org/10.1172/JCI118532

64. Verschuren L, Kleemann R, Offerman EH, Szalai AJ, Emeis SJ, Princen HM, Kooistra T (2005) Effect of low dose atorvastatin versus diet-induced cholesterol lowering on atherosclerotic lesion progression and inflammation in apolipoprotein $\mathrm{E}^{*} 3$-Leiden transgenic mice. Arterioscler Thromb Vasc Biol 25:161-167. https:// doi.org/10.1161/01.ATV.0000148866.29829.19

65. Weinberger T, Esfandyari D, Messerer D, Percin G, Schleifer C, Thaler R, Liu L, Stremmel C, Schneider V, Vagnozzi RJ, Schwanenkamp J, Fischer M, Busch K, Klapproth K, IshikawaAnkerhold H, Klösges L, Titova A, Molkentin JD, Kobayashi Y, Engelhardt S, Massberg S, Waskow C, Perdiguero EG, Schulz C (2020) Ontogeny of arterial macrophages defines their functions in homeostasis and inflammation. Nat Commun 11:4549. https:// doi.org/10.1038/s41467-020-18287-x

66. Weitz-Schmidt G, Welzenbach K, Brinkmann V, Kamata T, Kallen J, Bruns C, Cottens S, Takada Y, Hommel U (2001) Statins selectively inhibit leukocyte function antigen-1 by binding to a novel regulatory integrin site. Nat Med 7:687-692. https://doi. org/10.1038/89058

67. Williams JW, Martel C, Potteaux S, Esaulova E, Ingersoll MA, Elvington A, Saunders BT, Huang LH, Habenicht AJ, Zinselmeyer BH, Randolph GJ (2018) Limited macrophage positional dynamics in progressing or regressing murine atherosclerotic plaquesbrief report. Arterioscler Thromb Vasc Biol 38:1702-1710. https ://doi.org/10.1161/ATVBAHA.118.311319

68. Williams JW, Zaitsev K, Kim KW, Ivanov S, Saunders BT, Schrank PR, Kim K, Elvington A, Kim SH, Tucker CG, Wohltmann M, Fife BT, Epelman S, Artyomov MN, Lavine KJ, Zinselmeyer BH, Choi JH, Randolph GJ (2020) Limited proliferation capacity of aortic intima resident macrophages requires monocyte recruitment for atherosclerotic plaque progression. Nat Immunol 21:1194-1204. https://doi.org/10.1038/s41590-020-0768-4

69. Yin W, Carballo-Jane E, McLaren DG, Mendoza VH, Gagen K, Geoghagen NS, McNamara LA, Gorski JN, Eiermann GJ, Petrov A, Wolff M, Tong X, Wilsie LC, Akiyama TE, Chen J, Thankappan A, Xue J, Ping X, Andrews G, Wickham LA, Gai CL, Trinh T, Kulick AA, Donnelly MJ, Voronin GO, Rosa R, Cumiskey AM, Bekkari K, Mitnaul LJ, Puig O, Chen F, Raubertas R, Wong PH, Hansen BC, Koblan KS, Roddy TP, Hubbard BK, Strack AM (2012) Plasma lipid profiling across species for the identification of optimal animal models of human dyslipidemia. J Lipid Res 53:51-65. https://doi.org/10.1194/jlr.M019927

70. Zadelaar S, Kleemann R, Verschuren L, de Vries-Van der Weij J, van der Hoorn J, Princen HM, Kooistra T (2007) Mouse models for atherosclerosis and pharmaceutical modifiers. Arterioscler Thromb Vasc Biol 27:1706-1721. https://doi.org/10.1161/ ATVBAHA.107.142570

71. Zernecke A, Winkels H, Cochain C, Williams JW, Wolf D, Soehnlein O, Robbins CS, Monaco C, Park I, McNamara CA, Binder CJ, Cybulsky MI, Scipione CA, Hedrick CC, Galkina EV, Kyaw T, Ghosheh Y, Dinh HQ, Ley K (2020) Meta-analysis of leukocyte diversity in atherosclerotic mouse aortas. Circ Res 127:402-426. https://doi.org/10.1161/CIRCRESAHA.120.316903 